\title{
Continuous Maps from Spheres Converging to Boundaries of Convex Hulls
}

\author{
Joseph Malkoun ${ }^{\left(D_{1}\right.}$ and Peter J. Olver ${ }^{\left(D_{2}\right.}$ \\ ${ }^{1}$ Department of Mathematics and Statistics, Faculty of Natural and Applied Sciences, Notre Dame University-Louaize, \\ Zouk Mosbeh, P.O. Box 72, Zouk Mikael, Lebanon; E-mail: joseph.malkoun@ndu.edu.lb. \\ ${ }^{2}$ School of Mathematics, University of Minnesota, Minneapolis, MN 55455, USA; E-mail: olver@umn.edu.
}

Received: 21 July 2020; Revised: 21 December 2020; Accepted: 22 January 2021

2020 Mathematics Subject Classification: Primary - 52B55; Secondary - 26E25

\begin{abstract}
Given $n$ distinct points $\mathbf{x}_{1}, \ldots, \mathbf{x}_{n}$ in $\mathbb{R}^{d}$, let $K$ denote their convex hull, which we assume to be $d$-dimensional, and $B=\partial K$ its $(d-1)$-dimensional boundary. We construct an explicit, easily computable one-parameter family of continuous maps $\mathbf{f}_{\varepsilon}: \mathbb{S}^{d-1} \rightarrow K$ which, for $\varepsilon>0$, are defined on the $(d-1)$-dimensional sphere, and whose images $\mathbf{f}_{\mathcal{E}}\left(\mathbb{S}^{d-1}\right)$ are codimension 1 submanifolds contained in the interior of $K$. Moreover, as the parameter $\varepsilon$ goes to $0^{+}$, the images $\mathbf{f}_{\varepsilon}\left(\mathbb{S}^{d-1}\right)$ converge, as sets, to the boundary $B$ of the convex hull. We prove this theorem using techniques from convex geometry of (spherical) polytopes and set-valued homology. We further establish an interesting relationship with the Gauss map of the polytope $B$, appropriately defined. Several computer plots illustrating these results are included.
\end{abstract}

\section{Introduction}

Given a configuration $X=\left(\mathbf{x}_{1}, \ldots, \mathbf{x}_{n}\right)$ of $n$ distinct points in $\mathbb{R}^{d}$, computing their convex hull $K=$ $\operatorname{Conv}(X)$ is a famous problem in computational geometry. Many algorithms have been developed for this task, including the gift wrap or Jarvis march algorithm, the Graham scan algorithm, QuickHull, divide and conquer, the monotone chain or Andrew's algorithm, Chan's algorithm, the incremental convex hull algorithm, the ultimate planar convex hull algorithm and others (see, for instance, [3] and the references therein).

In this paper, we develop an alternative, direct approach to this problem that does not rely on any underlying computer algorithm. Instead, assuming $\operatorname{dim} K=d-$ meaning that its interior $K^{\circ}$ is a nonempty open subset of $\mathbb{R}^{d}$ - we construct a one-parameter family of approximations to its ( $d-1)$-dimensional boundary $B=\partial K$, which is a convex polytope, as the images of continuous maps $\mathbf{f}_{\varepsilon}: \mathbb{S}^{d-1} \rightarrow \mathbb{R}^{d}$ for $\varepsilon>0$, that are defined explicitly, and fairly simply, in terms of the points $\mathbf{x}_{1}, \ldots, \mathbf{x}_{n}$.

Initial computer-generated plots suggest that the images $\mathbf{f}_{\varepsilon}\left(\mathbb{S}^{d-1}\right)$ of our family of maps provide excellent approximations to the boundary $B$ for all configurations that we have tried; see Figures 1 and 2 for some representative examples. Our main result, Theorem 2.1, states that the images $\mathbf{f}_{\varepsilon}\left(\mathbb{S}^{d-1}\right)$ converge, as sets, to the boundary $B$ as the parameter $\varepsilon \rightarrow 0^{+}$. We will also explain in detail the mechanism of convergence. We then establish a relationship with the Gauss map of a smooth surface [8], thereby defining set-valued versions of the Gauss map and its inverse for the boundary of the convex hull. Indeed, our proof of the main theorem relies on techniques from the theory of set-valued homology.

(C) The Author(s), 2021. Published by Cambridge University Press. This is an Open Access article, distributed under the terms of the Creative Commons Attribution licence (http://creativecommons.org/licenses/by/4.0/), which permits unrestricted re-use, distribution, and reproduction in any medium, provided the original work is properly cited. The written permission of Cambridge University Press must be obtained for commercial re-use or in order to create a derivative work. 


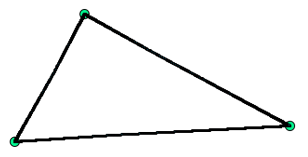

(a) $n=3$

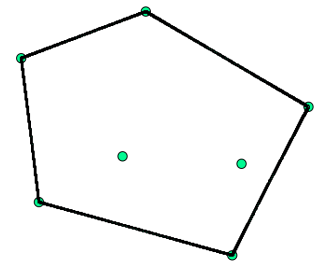

(d) $n=7$

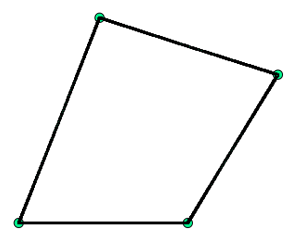

(b) $n=4$

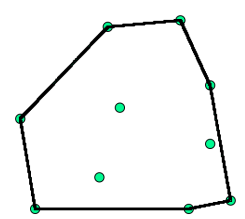

(e) $n=10$

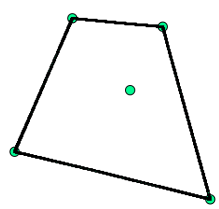

(c) $n=5$

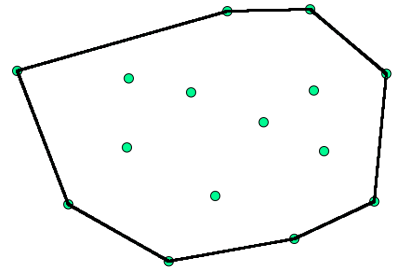

(f) $n=15$

Figure 1. Plots of the image $\mathbf{f}_{\varepsilon}\left(\mathbb{S}^{1}\right)$ with $\varepsilon=0.01$ for some planar point configurations $X$.

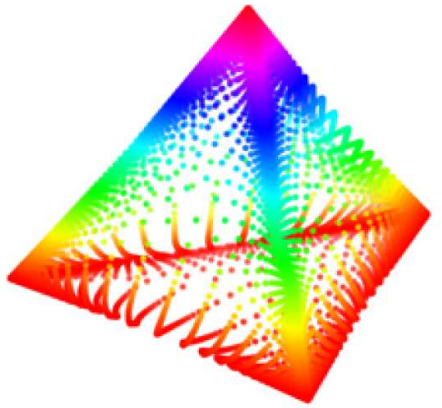

(a) tetrahedron

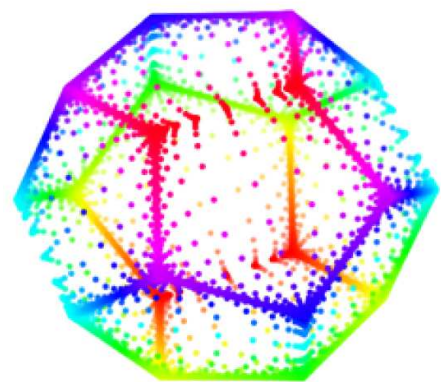

(c) dodecahedron

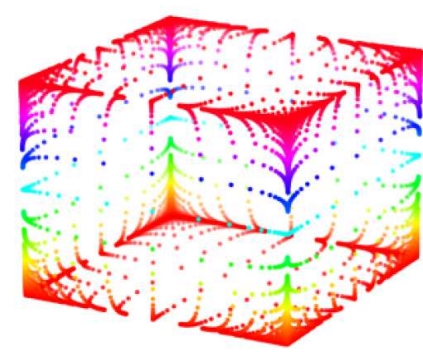

(b) cube

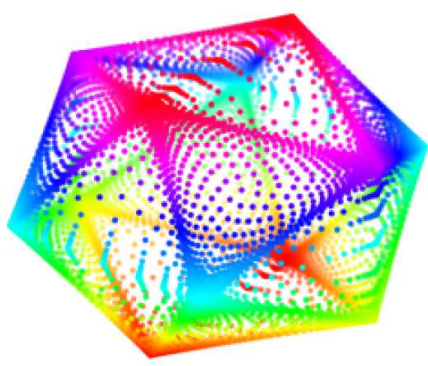

(d) icosahedron

Figure 2. Plots of the images of $\approx 15,000$ sample points on $\mathbb{S}^{2}$ under the map $\mathbf{f}_{\varepsilon}$, with $\varepsilon=0.1$ corresponding to point configurations $X$ consisting of the vertices of four of the regular polyhedra in dimension $d=3$. 
On the other hand, the convergence of the approximating sets $\mathbf{f}_{\varepsilon}\left(\mathbb{S}^{d-1}\right)$ to the boundary $B$ is highly nonuniform. Indeed, as we will see, the images $\mathbf{f}_{\varepsilon}(\mathbf{n})$ of almost every point $\mathbf{n} \in \mathbb{S}^{d-1}$ converge to one of the vertices of $B$. Thus, if one discretely samples $\mathbb{S}^{d-1}$ by a large but finite number of points $\mathbf{y}_{1}, \ldots, \mathbf{y}_{N}$, most of their image points $\mathbf{f}_{\varepsilon}\left(\mathbf{y}_{k}\right) \in \mathbf{f}_{\mathcal{\varepsilon}}\left(\mathbb{S}^{d-1}\right)$ will accumulate around the vertices of $B$, and the remainder of $B$ will be increasingly sparsely approximated as $\varepsilon \rightarrow 0^{+}$. This nonuniform sampling property can be observed in the three-dimensional illustrative plots in Figure 2.

The primary focus of this paper is to prove the convergence theorem and to establish interesting and potentially useful connections with convex analysis, with the geometry of polytopes and spherical polytopes and with the Gauss map from differential geometry. A future research project that has served to inspire these constructions will be the development of potential new practical algorithms for approximating or computing the convex hull of a point configuration. An interesting further extension of our techniques could be to the approximation of Wulff shapes of crystals [15].

Methods for approximating the boundary of convex polytopes by smooth submanifolds date back to Minkowski [14]; see [7] for more recent results and [6] for the construction of approximating algebraic sets. In these approaches, one is required to a priori know the facets of the polytope, whereas our method works directly on the point configurations themselves.

\section{A family of maps defined by a point configuration}

Let us begin by introducing the basic setup and our notation, before defining the family of maps that will be our primary object of study.

Let $C_{n}\left(\mathbb{R}^{d}\right)$ denote the configuration space of $n$ distinct points in $\mathbb{R}^{d}$; in other words, $X=$ $\left(\mathbf{x}_{1}, \ldots, \mathbf{x}_{n}\right) \in C_{n}\left(\mathbb{R}^{d}\right)$ means that each $\mathbf{x}_{i} \in \mathbb{R}^{d}$ and $\mathbf{x}_{i} \neq \mathbf{x}_{j}$ whenever $i \neq j$. Assuming $n \geq d+1$, let $C_{n}^{*}\left(\mathbb{R}^{d}\right) \subset C_{n}\left(\mathbb{R}^{d}\right)$ denote the dense open subset of nondegenerate configurations, meaning those whose points do not all lie on a proper affine subspace of $\mathbb{R}^{d}$. From here on we fix the nondegenerate point configuration $X \in C_{n}^{*}\left(\mathbb{R}^{d}\right)$ and suppress all dependencies thereon.

Let $K=\operatorname{Conv}(X) \subset \mathbb{R}^{d}$ denote the convex hull of the points in $X$, which by nondegeneracy is a bounded convex polytope of dimension $d$ whose interior is a nonempty open subset $K^{\circ} \subset \mathbb{R}^{d}[9,18]$. Let $B=\partial K=\partial \operatorname{Conv}(X)$ be its boundary, which is a closed polyhedral (piecewise linear) hypersurface in $\mathbb{R}^{d}$.

Let $\mathbb{R}^{+}=\{0<t \in \mathbb{R}\}$. Given any pair of indices $1 \leq i, j \leq n$ with $i \neq j$, we define real-valued functions $c_{i j}: \mathbb{R}^{+} \times \mathbb{S}^{d-1} \rightarrow \mathbb{R}^{+}$by

$$
c_{i j}(\varepsilon, \mathbf{n})=\varepsilon+\max \left\{0,-\left\langle\mathbf{n}, \mathbf{n}_{i j}\right\rangle\right\}, \quad \varepsilon>0, \quad \mathbf{n} \in \mathbb{S}^{d-1},
$$

where $\langle\cdot, \cdot\rangle$ denotes the Euclidean inner product in $\mathbb{R}^{d}$ and where

$$
\mathbf{n}_{i j}=\frac{\mathbf{x}_{j}-\mathbf{x}_{i}}{\left\|\mathbf{x}_{j}-\mathbf{x}_{i}\right\|} \in \mathbb{S}^{d-1}, \quad i \neq j,
$$

is the unit vector pointing from $\mathbf{x}_{i}$ to $\mathbf{x}_{j}$, with $\|\cdot\|$ denoting the Euclidean norm. Note that $\mathbf{n}_{i j}=-\mathbf{n}_{j i}$. The $c_{i j}$ in equation (2.1) are continuous maps; moreover, $c_{i j}(\varepsilon, \mathbf{n})>0$, since we are assuming (for now) that $\varepsilon>0$. We further define, for any $1 \leq i \leq n$, the map $c_{i}: \mathbb{R}^{+} \times \mathbb{S}^{d-1} \rightarrow \mathbb{R}^{+}$by the $(n-1)$-fold product

$$
c_{i}(\varepsilon, \mathbf{n})=\prod_{\substack{1 \leq j \leq n \\ j \neq i}} c_{i j}(\varepsilon, \mathbf{n}) .
$$

Finally, let us set

$$
\lambda_{i}(\varepsilon, \mathbf{n})=\frac{c_{i}(\varepsilon, \mathbf{n})}{\Delta(\varepsilon, \mathbf{n})}, \quad i=1, \ldots, n,
$$




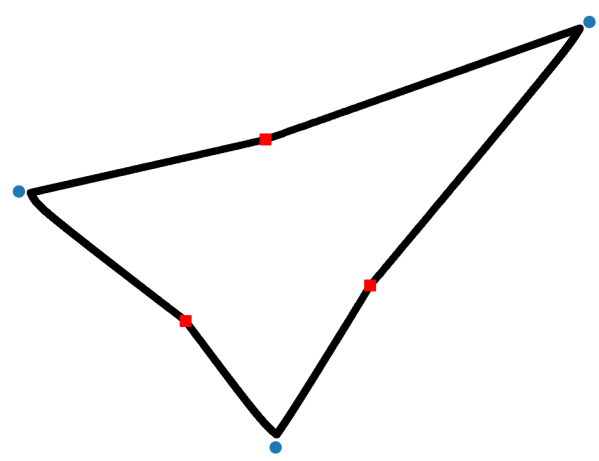

Figure 3. $X$ consists of the vertices of a triangle, with $\varepsilon=0.1$. One can see that $\mathbf{f}_{\varepsilon}\left(\mathbb{S}^{1}\right)$ is indented at the points $\mathbf{f}_{\varepsilon}(\mathbf{n})$, shown as small red squares, which correspond to $\mathbf{n} \in S_{i j}$ for $1 \leq i, j \leq 3$ and $i \neq j$.

where

$$
\Delta(\varepsilon, \mathbf{n})=\sum_{j=1}^{n} c_{j}(\varepsilon, \mathbf{n})>0 \quad \text { for all } \quad \varepsilon>0, \quad \mathbf{n} \in \mathbb{S}^{d-1}
$$

Thus,

$$
0<\lambda_{i}(\varepsilon, \mathbf{n})<1 \quad \text { and } \quad \sum_{i=1}^{n} \lambda_{i}(\varepsilon, \mathbf{n})=1 .
$$

Given a point configuration $X \in C_{n}\left(\mathbb{R}^{d}\right)$, we can now define the main object of interest in this paper: the one-parameter family of maps $\mathbf{f}_{\varepsilon}: \mathbb{S}^{d-1} \rightarrow \mathbb{R}^{d}$ defined by

$$
\mathbf{f}_{\varepsilon}(\mathbf{n})=\sum_{i=1}^{n} \lambda_{i}(\varepsilon, \mathbf{n}) \mathbf{x}_{i}, \quad \varepsilon>0, \quad \mathbf{n} \in \mathbb{S}^{d-1} .
$$

From formulas (2.6) and (2.7), one immediately deduces that

$$
\mathbf{f}_{\varepsilon}(\mathbf{n}) \in K^{\circ} \quad \text { for any } \quad \varepsilon>0, \quad \mathbf{n} \in \mathbb{S}^{d-1} .
$$

Inspection of Figures 1 and 2, and others that can be easily generated by computer, indicates that for a given $X \in C_{n}^{*}\left(\mathbb{R}^{d}\right)$ and small $\varepsilon>0$, the image of $\mathbb{S}^{d-1}$ under $\mathbf{f}_{\varepsilon}$ may be used as a good approximation of the boundary $B=\partial K \subset \mathbb{R}^{d}$ of the convex hull of $X$. More precisely, the main theorem to be proved in this paper is as follows:

Theorem 2.1. Given a nondegenerate point configuration $X \in C_{n}^{*}\left(\mathbb{R}^{d}\right)$, let $K=\operatorname{Conv}(X)$ be its convex hull, which has dimension $d$. Let $\mathbf{f}_{\varepsilon}$ be defined by equation (2.7). Then for $\varepsilon>0$, the images of the unit sphere under $\mathbf{f}_{\varepsilon}$ lie in the interior of the convex hull of $X$, so $\mathbf{f}_{\varepsilon}\left(\mathbb{S}^{d-1}\right) \subset K^{\circ}$ and, moreover, converge to its boundary as sets in $\mathbb{R}^{d}$ :

$$
\lim _{\varepsilon \rightarrow 0^{+}} \mathbf{f}_{\varepsilon}\left(\mathbb{S}^{d-1}\right)=\partial K
$$

The set-theoretic convergence in equation (2.8) is uniform in the sense that the images $\mathbf{f}_{\mathcal{E}}\left(\mathbb{S}^{d-1}\right)$ lie in an $\mathrm{O}(\varepsilon)$ neighbourhood of the boundary $\partial K$, even though their pointwise convergence is highly nonuniform. See later for precise details on what this means.

Remark. On the other hand, if the point configuration is degenerate - meaning $X \in C_{n}\left(\mathbb{R}^{d}\right) \backslash C_{n}^{*}\left(\mathbb{R}^{d}\right)$ - and so its convex hull $K=\operatorname{Conv}(X)$ has dimension strictly less than $d$, then one can show that $\lim _{\varepsilon \rightarrow 0^{+}} \mathbf{f}_{\varepsilon}\left(\mathbb{S}^{d-1}\right)=K$. Indeed, observe that the maps $\mathbf{f}_{\varepsilon}$ depend continuously on the point configuration. 
If one slightly perturbs $X$ to a nondegenerate configuration $X_{\delta} \in C_{n}^{*}\left(\mathbb{R}^{d}\right)$, then their perturbed convex hull $K_{\delta}$ is of dimension $d$ and, by Theorem 2.1, $\mathbf{f}_{\varepsilon}\left(\mathbb{S}^{d-1}\right) \rightarrow \partial K_{\delta}$. But as $\delta \rightarrow 0$, their boundaries converge to the entire convex hull: $\partial K_{\delta} \rightarrow K$, which enables one to establish the result. Since this case is of less importance for our purposes, the details are left to the reader.

Remark. In this paper, we have used the language of convergence of sequences (or nets) of compact subsets of an ambient metric space (upper limits and lower limits). According to [2], these notions originated with Painlevé in 1902, although they are usually named after Kuratowski, who popularised them in his book [10]. In our case, the ambient metric space is the convex hull $K=\operatorname{Conv}(X)$ of the points. Because $K$ is compact, the convergence of a sequence of compact subsets in the Kuratowski sense is equivalent to the convergence of the same sequence with respect to the Hausdorff metric. Thus, under the conditions of Theorem 2.1, our result also shows that the limit, in the Hausdorff metric, of $\mathbf{f}_{\varepsilon}\left(\mathbb{S}^{d-1}\right)$ as $\varepsilon \rightarrow 0$ is $\partial K$.

In Section 3, we present notions from convex geometry that are relevant to this work, including normal cones and normal spherical polytopes. The latter enable us to associate with a convex polytope $B$ a spherical complex $S_{B}^{*}$ [13], meaning a tiling of $\mathbb{S}^{d-1}$ by spherical polytopes, with the property that it has the same combinatorial type as the dual polytope $B^{*}$. Then in Section 5, we connect our constructions with the differential geometric concept of the Gauss map of a convex hypersurface, generalised to the boundary of the convex polytope. We explain how our maps converge to the inverse Gauss map of the boundary of the convex hull of the point configuration, which is viewed as a set-valued function. Finally, in Section 7, we prove our main result using a combination of convex geometry and set-valued homology theory, the latter described in Appendix A.

\section{Convex geometry, polytopes and spherical polytopes}

Let us recall some basic terminology and facts about convex sets and cones and both flat and spherical polytopes, many of which can be found in $[4,17]$. The closed cones appearing in this paper are convex; pointed, meaning they do not contain any positive dimensional linear subspace of $\mathbb{R}^{d}$; and polyhedral, meaning they can be characterised as the intersection of finitely many, and at least two, closed half spaces $[5,18]$. On the other hand, for us an open cone $N \subset \mathbb{R}^{d}$ is a cone such that $N \backslash\{0\}$ is an open subset of $\mathbb{R}^{d}$ and its closure $\bar{N}$ is of the earlier type.

Let us fix a nondegenerate point configuration $X \in C_{n}^{*}\left(\mathbb{R}^{d}\right)$ consisting of $n$ distinct points $\mathbf{x}_{1}, \ldots, \mathbf{x}_{n} \in \mathbb{R}^{d}$. Let $K=\operatorname{Conv}(X) \subset \mathbb{R}^{d}$ denote the convex hull of the points in $X$, which is a bounded convex $d$-dimensional polytope $[9,18]$. Let $B=\partial K=\partial \operatorname{Conv}(X)$ be its boundary, which is itself a polytope of dimension $d-1$, and hence a closed, convex polyhedral hypersurface in $\mathbb{R}^{d}$. Assume, by relabelling if necessary, that $\mathbf{x}_{1}, \ldots, \mathbf{x}_{\kappa}$ are the vertices of $K$, while $\mathbf{x}_{\kappa+1}, \ldots, \mathbf{x}_{n}$ are the remaining points, which may lie either in the interior $K^{\circ}$ or at a nonvertex point of the boundary $B$. The faces of $B$ range in dimension from 0 , the vertices, to 1 , the edges, up to $d-1$, the facets. Two vertices are adjacent if they are the endpoints of a common edge. Note that each face $F \subset K$ is itself a convex polytope. If $0<m \leq d-1$, we denote the interior of an $m$-dimensional face $F$ by $F^{\circ}=F \backslash \partial F$, which is a flat $m$-dimensional submanifold of $\mathbb{R}^{d}$. (Keep in mind that this is not the same as its interior as a subset of $\mathbb{R}^{d}$, which is empty.)

Define the normal cone at the point $\mathbf{x}_{i}$ by

$$
N_{i}=\left\{\mathbf{y} \in \mathbb{R}^{d} \mid\left\langle\mathbf{y}, \mathbf{n}_{i j}\right\rangle \leq 0 \text { for all } j \neq i\right\}=\bigcap_{j \neq i} H_{i j},
$$

where the unit vectors $\mathbf{n}_{i j} \in \mathbb{S}^{d-1}$ are given in equation (2.2) and

$$
H_{i j}=\left\{\mathbf{y} \in \mathbb{R}^{d} \mid\left\langle\mathbf{y}, \mathbf{n}_{i j}\right\rangle \leq 0\right\}
$$


is the closed half space opposite to $\mathbf{n}_{i j}$. Further, let

$$
N_{i}^{\circ}=\left\{\mathbf{y} \in \mathbb{R}^{d} \mid\left\langle\mathbf{y}, \mathbf{n}_{i j}\right\rangle<0 \text { for all } j \neq i\right\}
$$

denote the interior of the normal cone $N_{i}$. It is easy to see that $N_{i}^{\circ} \neq \varnothing$ if and only if $\mathbf{x}_{i}$ is a vertex. Also, $N_{i}^{\circ} \cap N_{j}^{\circ}=\varnothing$ whenever $i \neq j$. Indeed, if $\mathbf{y} \in N_{i}^{\circ}$, then $\left\langle\mathbf{y}, \mathbf{n}_{i j}\right\rangle<0$. But then $\left\langle\mathbf{y}, \mathbf{n}_{j i}\right\rangle=\left\langle\mathbf{y},-\mathbf{n}_{i j}\right\rangle>0$, and hence $\mathbf{y} \notin N_{j}^{\circ}$. Furthermore, the union of the vertex normal cones is the entire space:

$$
\bigcup_{i=1}^{\kappa} N_{i}=\mathbb{R}^{d}
$$

that is, every vector is in one of the normal cones. This is a direct consequence of the supporting hyperplane theorem (see, for instance, [4, pp. 50-51]).

A spherical polytope is characterised as the intersection of finitely many closed hemispheres that does not contain any antipodal points (compare [5, §2.2]). It can alternatively be characterised as the intersection $C \cap \mathbb{S}^{d-1}$ of the unit sphere with a pointed polyhedral cone $C \subset \mathbb{R}^{d}$. Let us consequently define the normal spherical polytope

$$
S_{i}=N_{i} \cap \mathbb{S}^{d-1}=\left\{\mathbf{n} \in \mathbb{S}^{d-1} \mid\left\langle\mathbf{n}, \mathbf{n}_{i j}\right\rangle \leq 0 \text { for all } j \neq i\right\},
$$

associated with the point $\mathbf{x}_{i}$. Its interior

$$
S_{i}^{\circ}=N_{i}^{\circ} \cap \mathbb{S}^{d-1}=\left\{\mathbf{n} \in \mathbb{S}^{d-1} \mid\left\langle\mathbf{n}, \mathbf{n}_{i j}\right\rangle<0 \text { for all } j \neq i\right\}
$$

is nonempty if and only if $\mathbf{x}_{i}$ is a vertex, in which case it is an open submanifold of the unit sphere. Note that, by equation (3.4) and the preceding remarks,

$$
\bigcup_{i=1}^{\kappa} S_{i}=\mathbb{S}^{d-1}, \quad S_{i}^{\circ} \cap S_{j}^{\circ}=\varnothing, \quad i \neq j .
$$

The normal cone and normal spherical polytope associated with a general point $\mathbf{x} \in K$ in the convex hull are similarly defined:

$$
\begin{aligned}
N_{\mathbf{x}} & =\left\{\mathbf{y} \in \mathbb{R}^{d} \mid\langle\mathbf{y}, \mathbf{z}-\mathbf{x}\rangle \leq 0 \text { for all } \mathbf{z} \in K\right\}, \\
S_{\mathbf{x}} & =N_{\mathbf{x}} \cap \mathbb{S}^{d-1}=\left\{\mathbf{n} \in \mathbb{S}^{d-1} \mid\langle\mathbf{n}, \mathbf{z}-\mathbf{x}\rangle \leq 0 \text { for all } \mathbf{z} \in K\right\} .
\end{aligned}
$$

As before, $N_{\mathbf{x}}=\{0\}$ if $\mathbf{x} \in K^{\circ}$, while $N_{\mathbf{x}_{i}}=N_{i}$ when $\mathbf{x}_{i}$ is a vertex. More generally, if $F \subset B=\partial K$ is an $m$-dimensional face, then the normal cone $N_{\mathbf{x}}$ is independent of the point $\mathbf{x} \in F^{\circ}$ lying in its interior, and we thus define $N_{F}=N_{\mathbf{x}}$ for any such $\mathbf{x} \in F^{\circ}$. If the face $F$ has dimension $m$, then $N_{F}$ is a $(d-m)$-dimensional cone. Define its interior to be $N_{F}^{\circ}=N_{F} \backslash \partial N_{F}$, which is a $(d-m)$-dimensional submanifold of $\mathbb{R}^{d}$. Warning: unless $m=0$, so that $F$ is a vertex, $N_{F}^{\circ}$ is not the same as the interior of $N_{F}$ considered as a subset of $\mathbb{R}^{d}$, which is empty. In particular, if $F$ is a facet - that is, a $(d-1)$ dimensional face - then $N_{F}$ is a one-dimensional cone - that is, a ray in the direction of its unit outward normal $\mathbf{n}_{F}$ - with $N_{F}^{\circ}=\left\{c \mathbf{n}_{F} \mid c>0\right\}$. Observe that if $H \subset \partial F$ is a subface, then $N_{F} \subset \partial N_{H}$. Further, the convexity of $K$ implies that $N_{F}^{\circ} \cap N_{G}^{\circ}=\varnothing$ whenever $F \neq G$ are distinct faces of $B$; in particular, $\mathbf{n}_{F} \neq \mathbf{n}_{G}$ whenever $F \neq G$ are distinct facets.

The collection of the interiors of all the normal cones to the faces of $B$ forms the complete normal fan associated with the polytopes $B$ and $K$, and their disjoint union fills out the entire space, except for the origin (which can be identified with $N_{K}$ ):

$$
\mathbb{R}^{d}=\{0\} \sqcup \bigsqcup_{F \subset B} N_{F}^{\circ},
$$

where we use the symbol $\sqcup$ to emphasise that the union is disjoint. 
We further define the normal spherical polytope associated with the $m$-dimensional face $F$ as $S_{F}=N_{F} \cap \mathbb{S}^{d-1}$. When $m<d-1$, its interior $S_{F}^{\circ}=N_{F}^{\circ} \cap \mathbb{S}^{d-1}$ is a $(d-m-1)$-dimensional submanifold of $\mathbb{S}^{d-1}$, while for $m=d-1$, the normal spherical polytope $S_{F}$ is a single point, namely the facet's unit outward normal $\mathbf{n}_{F}$. As an immediate consequence of the complete normal fan decomposition (3.9), we can write the sphere as a disjoint union

$$
\mathbb{S}^{d-1}=\bigsqcup_{\operatorname{dim} F<d-1} S_{F}^{\circ} \sqcup \bigsqcup_{\operatorname{dim} F=d-1} S_{F},
$$

where the second term runs over the facets and the first over all other faces of $B=\partial K$. As before, if $H \subset \partial F$ is a subface, then $S_{H} \subset \partial S_{G}$.

The collection of all normal spherical polytopes $S_{F}$, where $F$ runs over all faces of $B$, forms a spherical complex [13], denoted $S_{B}^{*}$, that tiles the sphere by spherical polytopes as shown in equation (3.10). We note that $S_{B}^{*}$ has the same combinatorial type as the dual polytope $B^{*}$ [9], and hence we regard the normal spherical complex $S_{B}^{*}$ as the spherical dual to $B$.

We can explicitly characterise where a given unit vector lies in this complex as follows. For $\mathbf{n} \in \mathbb{S}^{d-1}$ and $i=1, \ldots, n$, define

$$
\mu_{i}(\mathbf{n})=\min \left\{-\left\langle\mathbf{n}, \mathbf{n}_{i j}\right\rangle \mid 1 \leq j \leq n \text { and } j \neq i\right\} .
$$

According to equations (3.5) and (3.6), if $\mu_{i}(\mathbf{n})>0$, then $\mathbf{n} \in S_{i}^{\circ}$ and all other $\mu_{j}(\mathbf{n})<0$, while if

$$
\mathbf{n} \in T=\mathbb{S}^{d-1} \backslash S, \quad \text { where } \quad S=\bigsqcup_{i=1}^{k} S_{i}^{\circ},
$$

then all $\mu_{i}(\mathbf{n}) \leq 0$ for $i=1, \ldots, n$. In more detail:

Proposition 3.1. Let $\mathbf{n} \in \mathbb{S}^{d-1}$. Then

(i) $\mathbf{n} \in S_{i}^{\circ}$ for some $i=1, \ldots, n$ if and only if $\mu_{i}(\mathbf{n})>0$, while $\mu_{j}(n)<0$ for all $j \neq i$.

(ii) $\mathbf{n} \in S_{F}^{\circ}$ for some face $F$ with $0<\operatorname{dim} F<d-1$ or $\mathbf{n} \in S_{F}$ for some facet $F$ if and only if $\mu_{i}(\mathbf{n}) \leq 0$ for all $i=1, \ldots, n$ and $\mu_{j}(\mathbf{n})=0$ whenever $\mathbf{x}_{j} \in F$. In other words, $F$ is the convex hull of the points $\mathbf{x}_{j}$ for which $\mu_{j}(\mathbf{n})=0$.

Proof. We already established (i). As for (ii), we need only note that if $\mathbf{x}_{i}, \mathbf{x}_{j} \in F$ and $\mathbf{n}$ is normal to $F$, then $\left\langle\mathbf{n}, \mathbf{n}_{i j}\right\rangle=0$.

Remark. Maehara and Martini [12] propose a similar construction, which they call the 'outer normal transform' of a convex polytope $B \subset \mathbb{R}^{d}$ of dimension $d-1$. They associate each facet $F \subset B$ with its outward normal $\mathbf{n}_{F} \in \mathbb{S}^{d-1} \subset \mathbb{R}^{d}$. The outer normal transform of $B$ is defined to be the convex hull of the facet normals in $\mathbb{R}^{d}$. They observe that unlike the spherical dual, their transform is not necessarily combinatorially equivalent to the dual polytope $B^{*}$.

On the other hand, if we flatten all the normal spherical polytopes of the spherical dual $S_{B}^{*}$, meaning we replace each $S_{F} \subset \mathbb{R}^{d}$ by the convex hull of its vertices, the result will be a polytope $\widehat{B} \subset \mathbb{R}^{d}$ contained within the unit ball, all of whose vertices lie on the unit sphere. Although the resulting polytope $\widehat{B}$ also has the same combinatorial type as $B^{*}$, it is not necessarily convex. The outer normal transform of $B$ can thus be identified with the convex hull of $\widehat{B}$ and so, when $\widehat{B}$ is not convex, will possess a different combinatorial structure to $B^{*}$.

Indeed, counterexamples to the problem of inscribing convex polytopes of a given combinatorial type in spheres [16] are of this form. For example, the dual to the truncated tetrahedron, known as the triakis tetrahedron, ${ }^{1}$ is not inscribable in a sphere; see Figure 4 . The flattened version of the spherical dual to

\footnotetext{
${ }^{1}$ A triakis tetrahedron is obtained from a regular tetrahedron by gluing a simplex to each of its four faces. If the altitudes of the simplices are sufficiently short, the result is a convex polyhedron with 12 triangular faces.
} 


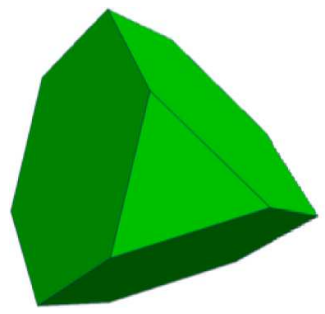

(a) Truncated tetrahedron

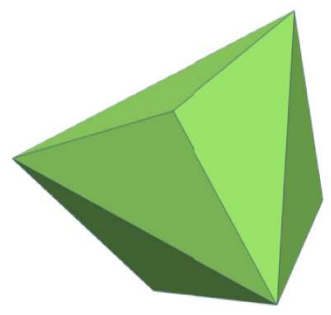

(b) Triakis tetrahedron

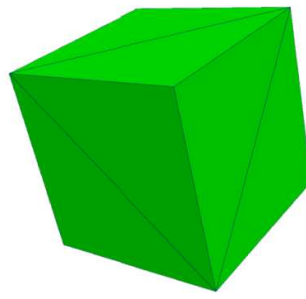

(c) Cubical triakis tetrahedron

Figure 4. Polytopes.

a truncated tetrahedron is a cube with diagonals that bisect each square into a pair of triangular facets and form the edges of an interior tetrahedron. Both the spherical dual and the resulting flattened cube with diagonals have the same combinatorial type as the triakis tetrahedron. However, the cubical triakis tetrahedron, while inscribed in the unit sphere, is not a convex polyhedron, since it has pairs of coplanar triangular facets possessing a common normal. It is, of course, the set-theoretic boundary of a convex subset of $\mathbb{R}^{3}$, namely the inscribed solid cube, whose cubical boundary (without the diagonals) can be identified as the outer normal transform of the original truncated tetrahedron, and is not combinatorially equivalent to the triakis tetrahedron. Furthermore, slightly perturbing the original truncated tetrahedron leads to a perturbed spherical dual and a perturbed cube with diagonals that is inscribed in the sphere, again both having the same combinatorial type as the triakis tetrahedron. However, although its triangular faces are no longer coplanar, the resulting polyhedron is not the boundary of a convex subset of $\mathbb{R}^{3}$, and hence not equal to its outer normal transform, which is the convex hull of this nonconvex perturbed cube. All this is a necessary consequence of the noninscribability of the triakis tetrahedron.

In general, if the flattened spherical dual of a polytope is convex, then it has to coincide with its outer normal transform, which is then, by the foregoing remarks, combinatorially equivalent to the dual polytope. On the other hand, if it is not convex, then its convexification, which is the outer normal transform, cannot be combinatorially equivalent to the dual. Thus we have established the following:

Proposition 3.2. Let $B \subset \mathbb{R}^{d}$ be a convex polytope of dimension $d-1$. Then the outer normal transform of $B$ is combinatorially equivalent to the dual polytope $B^{*}$ if and only if the flattened spherical dual of $B$ is convex.

Finally, for later purposes, we will introduce some useful open subsets of the normal spherical complex (3.10). If $F \subset B$ is a facet with outward unit normal $\mathbf{n}_{F} \in \mathbb{S}^{d-1}$, so that $S_{F}=\left\{\mathbf{n}_{F}\right\}$, set

$$
W_{F}=S_{F} \sqcup \bigsqcup_{G \subsetneq F} S_{G}^{\circ},
$$

where the union is over the proper subfaces $G \subsetneq F$. On the other hand, if $F \subset B$ is a face with $1 \leq \operatorname{dim} F<d-1$, set

$$
W_{F}=\bigsqcup_{G \subseteq F} S_{G}^{\circ} .
$$

Lemma 3.3. Under the foregoing definitions, $W_{F}$ is a relatively open subset of $\mathbb{S}^{d-1}$.

Proof. This follows from the fact that the corresponding union of normal cones

$$
V_{F}=\{0\} \sqcup \bigsqcup_{G \subseteq F} N_{G}^{\circ}
$$


is an open cone and $W_{F}=V_{F} \cap \mathbb{S}^{d-1}$. Indeed, one can use a perturbed version of the supporting hyperplane theorem that says that if $H$ is a supporting hyperplane such that $H \cap B=F$, where $F$ is a face, and $\widetilde{H}$ is a supporting hyperplane that is a sufficiently small perturbation of $H$, then $\widetilde{H} \cap B=G$ for some subface $G \subseteq F$. Keep in mind that the subface could be a vertex.

The last result of this section is a technical construction, which is key to our proof of theorem 2.1. The reader may wish to skip it for now and return once the proof is underway.

Proposition 3.4. Let $F \subset B$ be a face of dimension $1 \leq m \leq d-1$. Let $S_{F}$ be its normal spherical polytope and $W_{F} \subset \mathbb{S}^{d-1}$ be the open subset given by Lemma 3.3. Let $G_{1}, \ldots, G_{k}$ be its $(m-1)$ dimensional subfaces, so that $\partial F=\bigcup_{i=1}^{k} G_{i}$. Suppose $N \subset W_{F} \subset \mathbb{S}^{d-1}$ is a connected m-dimensional submanifold such that either (a) if $F$ is a facet, of dimension $d-1$, with unit outward normal $\mathbf{n}_{0}=\mathbf{n}_{F}$, then $N$ is an open neighbourhood of $\mathbf{n}_{0}$; or (b) if $1 \leq m=\operatorname{dim} F<d-1$, then $N$ intersects $S_{F}^{\circ}$ transversally at a single point $\mathbf{n}_{0} \in N \cap S_{F}^{\circ}$. Then if $\widetilde{N} \subset N$ is a sufficiently small open contractible submanifold with $\mathbf{n}_{0} \in \widetilde{N}$, which implies $\mathbf{n}_{0} \in \partial\left(\widetilde{N} \cap S_{G_{i}}^{\circ}\right)$ for all $i=1, \ldots, k$, we can decompose its boundary $\partial \widetilde{N}=\bigcup_{i=1}^{k} L_{i}$ into the union of $(m-1)$-dimensional submanifolds that only overlap on their boundaries, meaning $\bar{L}_{i} \cap \bar{L}_{j}=\partial L_{i} \cap \partial L_{j}$ whenever $i \neq j$, with the property that each $L_{i} \subset W_{G_{i}}$ intersects $S_{G_{i}}^{\circ}$ transversally at a single point $\mathbf{n}_{i} \in L_{i} \cap S_{G_{i}}^{\circ}=\partial \widetilde{N} \cap S_{G_{i}}^{\circ}$.

Proof. Choose $r>0$ sufficiently small that the relatively open submanifold $N_{r}=\left\{\mathbf{n} \in N \mid\left\|\mathbf{n}-\mathbf{n}_{0}\right\|<r\right\}$ has boundary $\partial N_{r}=\left\{\mathbf{n} \in N \mid\left\|\mathbf{n}-\mathbf{n}_{0}\right\|=r\right\}$. Moreover, reducing $r$ if necessary, we claim that $\partial N_{r}$ intersects each $S_{G_{i}}^{\circ}$ transversally at a single point $\mathbf{n}_{i} \in \partial N_{r} \cap S_{G_{i}}^{\circ}$. Indeed, in a small neighbourhood $\mathbf{n}_{0} \in U$ we can choose local coordinates centred at $\mathbf{n}_{0}$ such that, locally, $S_{F}^{\circ} \cap U$ is a $(d-m-1)$ dimensional subspace, $N_{r} \subset U$ is a transverse $m$-dimensional subspace and $S_{G_{i}}^{\circ} \cap U$ is a $(d-m)$ dimensional half space with local boundary $\partial S_{G_{i}} \cap U=S_{F}^{\circ} \cap U$, from which the preceding claim is evident.

We now set $\widetilde{N}=N_{r}$. Since $S_{F} \subset \partial S_{G_{i}}$, this immediately implies $\mathbf{n}_{0} \in \partial\left(\widetilde{N} \cap S_{G_{i}}^{\circ}\right)$. The final task is to decompose $\partial \widetilde{N}=\bigcup_{i=1}^{k} L_{i}$ as in the statement of the proposition. It is reasonably clear that there are many ways to do this, but for definiteness, here is one possible construction. First we note that by equations (3.13) and (3.14), either

$$
W_{F} \backslash\left\{\mathbf{n}_{F}\right\}=\bigcup_{i=1}^{k} W_{i} \quad \text { or } \quad W_{F} \backslash S_{F}^{\circ}=\bigcup_{i=1}^{k} W_{i},
$$

according to whether $F$ is a facet or not. We thus, for each $i=1, \ldots, k$, need to choose $L_{i} \subset \widetilde{N} \cap W_{i}$ with the requisite properties.

First, define the closed subset $\bar{L}_{i} \subset \partial \widetilde{N}$ to be the set of all $\mathbf{n} \in \widetilde{N} \cap W_{i}$ such that if $\mathbf{n} \in \widetilde{N} \cap S_{H}^{\circ}$ for some adjacent subface $H \subsetneq G_{i}$, then $\operatorname{dist}\left(\mathbf{n}, \mathbf{n}_{i}\right) \leq \operatorname{dist}\left(\mathbf{n}, \mathbf{n}_{j}\right)$ for all other adjacent $(m-1)$-dimensional subfaces $G_{j}$, meaning that $H \subsetneq G_{j}$. Clearly $N=\bigcup \bar{L}_{i}$, and moreover, $\bar{L}_{i}$ and $\bar{L}_{j}$ only overlap on their common boundary, which could be either part of a boundary of an $S_{H}$ or a point $\mathbf{n} \in N \cap S_{H}^{\circ}$ that is equidistant to $\mathbf{n}_{i}$ and $\mathbf{n}_{j}$. We then set $L_{i}=\bar{L}_{i}^{\circ}$ to be its interior ${ }^{2}$ relative to $\partial \widetilde{N}$. Since $\mathbf{n}_{i} \in L_{i}^{\circ}$, the transversality of $\partial \widetilde{N}$ to $S_{G_{i}}^{\circ}$ at $\mathbf{n}_{i}$ immediately implies the same for the relatively open submanifold $L_{i}$. We conclude that the resulting submanifolds satisfy the required conditions.

\footnotetext{
${ }^{2}$ It may happen that the closure of $L_{i}$ is strictly contained in $\bar{L}_{i}$; this can occur if there exist $\mathbf{n}_{l}$ associated with nonadjacent faces $G_{l}$ that lie closer to the points $\mathbf{n} \in \widetilde{N} \cap S_{H}^{\circ}$ than those in any adjacent face $G_{i}$. But this does not affect the construction, since every point in $\bar{L}_{i} \backslash L_{i}$ is contained in the boundary of some $L_{j}$.
} 


\section{Set-valued functions}

We now introduce an important generalisation of the notion of a function (see [1,2] for details). A setvalued function, also known as a multi-valued function, from a space $D$ to a space $Y$ means a mapping ${ }^{3}$ $\widehat{f}$ from $D$ to the power set $2^{Y}-$ that is, the set of subsets of $Y$. In other words, the image of $x \in D$ is a subset $\widehat{f}(x) \subset Y$. More generally, a set-valued function maps subsets of its domain to subsets of its range in the evident manner. We say that $\widehat{f}$ has closed values if $\widehat{f}(x)$ is a closed subset of $Y$ for all $x \in D$. The range $R \subset Y$ of $\widehat{f}$ is the union of all the images of points in its domain $D$, so $R=\widehat{f}(D)$. In particular, any ordinary function $f: D \rightarrow Y$ can be viewed as a set-valued function, with closed values, by identifying the image $y=f(x)$ of a point $x \in D$ with the singleton set $\{y\} \subset Y$.

In convex analysis, the normal cone (3.8) is often viewed as a set-valued function $\widehat{f}$ from $B=\partial K$ to $\mathbb{R}^{d}$ that maps a point $\mathbf{x} \in B$ to its normal cone $\widehat{f}(\mathbf{x})=N_{\mathbf{x}} \subset \mathbb{R}^{d}$. (One can, of course, extend it to all of $K$, but the values on the interior $K^{\circ}$ are trivial.) Similarly, we can view the normal spherical polytope construction as a set-valued function $\widehat{\gamma}_{B}$ from $B$ to $\mathbb{S}^{d-1}$ mapping a point $\mathbf{x} \in B$ to its normal spherical polytope: $\widehat{\gamma}_{B}(\mathbf{x})=S_{\mathbf{x}} \subset \mathbb{S}^{d-1}$.

We will be interested in the convergence of set-valued functions. Here is a simple example:

Example 4.1. Consider the ordinary functions

$$
f_{\varepsilon}(x)=\frac{2}{\pi}(1-\varepsilon) \arctan \frac{x}{\varepsilon} \quad \text { for } \quad x \in \mathbb{R}, \quad \varepsilon>0 .
$$

In the usual function-theoretic sense of convergence,

$$
\lim _{\varepsilon \rightarrow 0^{+}} f_{\varepsilon}(x)=\operatorname{sign} x= \begin{cases}1, & x>0 \\ 0, & x=0 \\ -1, & x<0\end{cases}
$$

Thus, for almost every point $x \in \mathbb{R}$, the value of $f_{\varepsilon}(x)$ converges to either -1 or 1 . However, if you look at their graphs as subsets of $\mathbb{R}^{2}$, they converge, as sets, to the curve consisting of the union of the three line segments

$$
\{(x,-1) \mid x \leq 0\} \cup\{(0, y) \mid-1 \leq y \leq 1\} \cup\{(x, 1) \mid x \geq 0\} .
$$

We can interpret this curve as the graph of the set-valued function

$$
\widehat{f}: \mathbb{R} \longrightarrow 2^{\mathbb{R}} \quad \text { given by } \quad \widehat{f}(x)= \begin{cases}\{1\}, & x>0, \\ {[-1,1],} & x=0, \\ \{-1\}, & x<0 .\end{cases}
$$

The domain of $\widehat{f}$ is $D=\mathbb{R}$ and its range is the interval $R=[-1,1]=\widehat{f}(\mathbb{R})$. Furthermore, the ranges $R_{\varepsilon}=f_{\varepsilon}(\mathbb{R})=(-1+\varepsilon, 1-\varepsilon)$ of the functions (4.1) are open intervals that converge, as sets, to the closed interval $[-1,1]$ forming the range of the limiting set-valued function.

In general, given spaces $D, R$, let $D \times R$ denote their Cartesian product and $\pi_{D}: D \times R \rightarrow D$ and $\pi_{R}: D \times R \rightarrow R$ the standard projections. Any subset $S \subset D \times R$ which projects onto both $D=\pi_{D}(S)$ and $R=\pi_{R}(S)$ defines a set-valued mapping $\widehat{f}$ with domain $D$ and range $R$, given by $\widehat{f}(x)=\pi_{R}\left(S \cap \pi_{D}^{-1}\{x\}\right)$. Its inverse $\widehat{f}^{-1}$ is also a set-valued mapping from $R$ to $D$, given by 
$\widehat{f}^{-1}(y)=\pi_{D}\left(S \cap \pi_{R}^{-1}\{y\}\right)$ [2]. For the above example (4.2), $\widehat{f}^{-1}:[-1,1] \rightarrow 2^{\mathbb{R}}$ is given by

$$
\widehat{f}^{-1}(y)= \begin{cases}(-\infty, 0], & y=-1, \\ \{0\}, & -1<y<1, \\ {[0, \infty),} & y=1 .\end{cases}
$$

Note that any ordinary function thus has a set-valued inverse.

Finally, we note that the concept of continuity does not extend straightforwardly to set-valued functions. The most important analogue is contained in the following definition:

Definition 4.2. Let $D$ and $R$ be topological spaces. A set-valued function $\widehat{f}: D \rightarrow R$ is called upper hemicontinuous at $x_{0} \in D$ if and only if for any open neighbourhood $V$ of the set $\widehat{f}\left(x_{0}\right)$, there exists a neighbourhood $U$ of $x_{0}$ such that $\widehat{f}(x) \subset V$ for all $x \in U$. We say that $\widehat{f}$ is upper hemicontinuous if it is upper hemicontinuous at every $x_{0} \in D$.

It is straightforward to verify that Example 4.1 satisfies the upper hemicontinuity condition.

Warning: A few authors, including [2], use the expression 'upper semicontinuous' instead of 'upper hemicontinuous'. However, the latter terminology seems to be more accepted by the broader community, particularly as it is not in conflict with the notion of semicontinuity of ordinary functions.

\section{The Gauss map of a convex polytope}

Let us now identify the preceding constructions with a set-valued version of the Gauss map of a hypersurface. Recall [8] that the Gauss map of a smooth closed hypersurface - that is, a $(d-1)$ dimensional oriented submanifold $M \subset \mathbb{R}^{d}-$ is defined as

$$
\gamma_{M}: M \longrightarrow \mathbb{S}^{d-1}, \quad \gamma(\mathbf{y})=\mathbf{n}_{\mathbf{y}}, \quad \mathbf{y} \in M,
$$

where $\mathbf{n}_{\mathbf{y}}$ denotes the unit outward normal to $M$ at $\mathbf{y}$. If $M$ is strictly convex, then its Gauss map is one-to-one and onto, with smooth inverse $\gamma_{M}^{-1}: \mathbb{S}^{d-1} \rightarrow M$.

We can identify the normal spherical polytope map $\widehat{\gamma}_{B}$ associated with a closed convex polyhedral hypersurface $B \subset \mathbb{R}^{d}$ (a convex polytope) as its set-valued Gauss map. If $M_{\varepsilon}$ for $\varepsilon>0$ are a parametrised family of smooth closed convex hypersurfaces converging uniformly to the polytope, $M_{\varepsilon} \rightarrow B$ as $\varepsilon \rightarrow 0^{+}$, then under suitable conditions, we would expect their Gauss maps $\gamma_{M_{\varepsilon}} \rightarrow \widehat{\gamma}_{B}$ to converge, in the sense of set-valued functions, to the set-valued normal spherical polytope map. Since this is not central to our results, we will not pursue this idea further here.

As for our construction, Theorem 2.1 shows that the functions $\mathbf{f}_{\varepsilon}: \mathbb{S}^{d-1} \rightarrow \mathbb{R}^{d}$ converge, in the set-valued sense, to a limiting set-valued function $\widehat{\mathbf{f}}_{0}: \mathbb{S}^{d-1} \rightarrow B$ defined as follows:

$$
\widehat{\mathbf{f}}_{0}(\mathbf{n})= \begin{cases}\mathbf{x}_{i}, & \mathbf{n} \in S_{i}^{\circ}, \\ F, & \mathbf{n} \in S_{F}^{\circ}, 0<\operatorname{dim} F<d-1, \\ & \text { or } \mathbf{n} \in S_{F}, \operatorname{dim} F=d-1 .\end{cases}
$$

Here $F$ refers to the various faces of $B$. Proposition 3.1 gives an explicit characterisation of the normal spherical complex sets $S_{F}, S_{F}^{\circ} \subset \mathbb{S}^{d-1}$ (compare equation (3.10)). The fact that the set-valued mapping $\widehat{\mathbf{f}}_{0}$ is the set-valued limit of $\mathbf{f}_{\varepsilon}$ as $\varepsilon \rightarrow 0^{+}$will follow from the proof of Theorem 2.1.

Moreover, we can identify the set-valued map (5.2) with the set-valued inverse of the Gauss map associated with the boundary of the convex hull: $\widehat{\mathbf{f}}_{0}=\widehat{\gamma}_{B}^{-1}$. On the other hand, the $\mathbf{f}_{\varepsilon}$ are certainly not inverse Gauss maps themselves. Moreover, simple examples, such as in Figure 3, show that the image $\mathbf{f}_{\varepsilon}\left(\mathbb{S}^{d-1}\right)$ is not in general a convex hypersurface. On the other hand, it might be worth investigating the set-theoretic convergence of their possibly multivalued Gauss maps. 


\section{Some computational lemmas}

Before launching into the proof of Theorem 2.1, let us collect together some elementary computational lemmas for the functions used to form the maps $\mathbf{f}_{\varepsilon}$ defined in equation (2.7).

Recalling equations (2.1) and (2.3), let us set

$$
c_{i j}(\mathbf{n})=\lim _{\varepsilon \rightarrow 0^{+}} c_{i j}(\varepsilon, \mathbf{n})=\max \left\{0,-\left\langle\mathbf{n}, \mathbf{n}_{i j}\right\rangle\right\}, \quad \mathbf{n} \in \mathbb{S}^{d-1}
$$

and

$$
c_{i}(\mathbf{n})=\lim _{\varepsilon \rightarrow 0^{+}} c_{i}(\varepsilon, \mathbf{n})=\prod_{\substack{1 \leq j \leq n \\ j \neq i}} c_{i j}(\mathbf{n}), \quad \mathbf{n} \in \mathbb{S}^{d-1} .
$$

We can thus write

$$
c_{i}(\varepsilon, \mathbf{n})=c_{i}(\mathbf{n})+\varepsilon b_{i}(\varepsilon, \mathbf{n}),
$$

where $b_{i}$ is a polynomial in $\varepsilon$ of degree $n-2$. In view of equations (3.6) and (3.7), $c_{i}(\mathbf{n})>0$ if and only if $\mathbf{n} \in S_{i}^{\circ}$, whereas $c_{i}(\mathbf{n})=0$ for all $i=1, \ldots, n$ if and only if $\mathbf{n} \in T$, the complement of the union of the $S_{i}^{\circ}$ (compare equation (3.12)). We have thus established the following result:

Lemma 6.1. Given $\mathbf{n} \in \mathbb{S}^{d-1}$, either all $c_{i}(\mathbf{n})=0$ or precisely one $c_{i}(\mathbf{n})>0$ and the rest are all zero. Moreover, in the latter case, $\mathbf{x}_{i}$ is a vertex.

Indeed, if $\mathbf{n} \in S_{i}^{\circ}$, then, referring to equations (2.3) and (6.3), $c_{i}(\mathbf{n})>0$ and $b_{i}(\varepsilon, \mathbf{n})>0$ for all $\varepsilon>0$, whereas

$$
c_{j}(\varepsilon, \mathbf{n})=\varepsilon^{1+k_{j}} a_{j}(\varepsilon, \mathbf{n}) \text { for } j \neq i,
$$

with $a_{j}(\varepsilon, \mathbf{n})>0$ for $\varepsilon>0$. The nonnegative integer $k_{j} \geq 0$ denotes the number of points $\mathbf{x}_{k}$ with $k \neq i, j$ that satisfy the distance inequality $\operatorname{dist}\left(\mathbf{x}_{k}, P_{i}(\mathbf{n})\right) \leq \operatorname{dist}\left(\mathbf{x}_{j}, P_{i}(\mathbf{n})\right)$, where $P_{i}(\mathbf{n})=\mathbf{x}_{i}+\mathbf{n}^{\perp}$, with $\mathbf{n}^{\perp}=\left\{\mathbf{y} \in \mathbb{R}^{d} \mid\langle\mathbf{y}, \mathbf{n}\rangle=0\right\}$ denoting the affine hyperplane orthogonal to $\mathbf{n}$ passing through $\mathbf{x}_{i}$. Note that there is either one or no value of $j$ for which $k_{j}=0$.

Let us finish this section by establishing a more detailed version of Lemma 6.1, valid for an arbitrary face $F \subset B$.

Lemma 6.2. Let $F \subset B$ be a face of dimension $1 \leq m \leq d-1$, with vertices $\mathbf{x}_{1}, \ldots, \mathbf{x}_{k}$. Suppose $F$ contains l additional nonvertex points $\mathbf{x}_{k+1}, \ldots, \mathbf{x}_{k+l}$, where l may be zero. Then, given $\mathbf{n} \in S_{F}^{\circ}$,

$$
\begin{array}{llrl}
c_{i}(\varepsilon, \mathbf{n}) & =\varepsilon^{k+l-1} d_{i}(\mathbf{n})+\varepsilon^{k+l} p_{i}(\varepsilon, \mathbf{n}), & & i=1, \ldots, k+l, \\
c_{j}(\varepsilon, \mathbf{n})=\varepsilon^{k+l} p_{j}(\varepsilon, \mathbf{n}), & j=k+l+1, \ldots, n,
\end{array}
$$

where $d_{i}(\mathbf{n})>0$, while $p_{1}(\varepsilon, \mathbf{n}), \ldots, p_{n}(\varepsilon, \mathbf{n})$ are polynomials in $\varepsilon$.

Proof. Let $\mathbf{n} \in S_{F}^{\circ}$. Note that $c_{i j}(\varepsilon, \mathbf{n})=\varepsilon$ whenever $\mathbf{x}_{i}, \mathbf{x}_{j} \in F$, so that $\left\langle\mathbf{n}, \mathbf{n}_{i j}\right\rangle=0$. On the other hand, $\left\langle\mathbf{n}, \mathbf{n}_{i j}\right\rangle<0$ whenever $\mathbf{x}_{i} \in F$ and $\mathbf{x}_{j} \notin F$, which, vice versa, implies $c_{j i}(\varepsilon, \mathbf{n})=\varepsilon$. The proof is completed by recalling the definition (2.3) of $c_{i}(\varepsilon, \mathbf{n})$.

\section{Proof of the Main Theorem}

Now we turn to the proof of theorem 2.1, on the convergence, as $\varepsilon \rightarrow 0^{+}$, of the hypersurfaces $\mathbf{f}_{\varepsilon}\left(\mathbb{S}^{d-1}\right) \subset K^{\circ} \subset \mathbb{R}^{d}$ to the boundary of the convex hull $B=\partial K$ of the point configuration $X$.

If we formally set $\varepsilon=0$ in the preceding definition (2.7) of the map $\mathbf{f}_{\varepsilon}$, Lemma 6.1 implies

$$
\lim _{\varepsilon \rightarrow 0^{+}} \mathbf{f}_{\varepsilon}(\mathbf{n})= \begin{cases}\mathbf{x}_{i}, & \mathbf{n} \in S_{i}^{\circ}, \\ \text { undefined, } & \mathbf{n} \in T=\mathbb{S}^{d-1} \backslash \bigsqcup_{i=1}^{K} S_{i}^{\circ} .\end{cases}
$$


Thus, for almost every point $\mathbf{n} \in \mathbb{S}^{d-1}$, the images $\mathbf{f}_{\varepsilon}(\mathbf{n})$ converge to one of the vertices of the convex hull. However, as in Example 4.1, this does not imply that, as a set, $\mathbf{f}_{\varepsilon}\left(\mathbb{S}^{d-1}\right)$ converges to the set of vertices $V=\left\{\mathbf{x}_{1}, \ldots, \mathbf{x}_{K}\right\}$. Our goal is to prove that the images $\mathbf{f}_{\varepsilon}\left(\mathbb{S}^{d-1}\right)$ converge, as sets, to the entire boundary $B$ as $\varepsilon \rightarrow 0^{+}$. Specifically, we will show the following:

○ Given any neighbourhood $W \supset B$, no matter how small, we can find $\varepsilon_{0}>0$ such that $\mathbf{f}_{\varepsilon}\left(\mathbb{S}^{d-1}\right) \subset W$ for any $0<\varepsilon<\varepsilon_{0}$.

$\circ$ Given any $\mathbf{x} \in B$, there exist points $\mathbf{y}_{\varepsilon} \in \mathbf{f}_{\varepsilon}\left(\mathbb{S}^{d-1}\right)$ for $\varepsilon>0$ such that $\mathbf{y}_{\varepsilon} \rightarrow \mathbf{x}$ as $\varepsilon \rightarrow 0^{+}$.

In the language of set-theoretic limits [2], the first statement shows that the outer limit of the sets $\mathbf{f}_{\mathcal{E}}\left(\mathbb{S}^{d-1}\right)$ is a subset of $B$. The second statement proves that $B$ is a subset of their inner limit. Since the inner limit is always a subset of the outer limit, this then implies that the inner and outer limits coincide and are equal to $B$.

First, recall that for $r>0$, the $r$-neighbourhood $U_{r}$ of a subset $D \subset \mathbb{R}^{d}$ is the set of points that are a distance less than $r$ (in the Euclidean norm) from $D$ - that is, $U_{r}=\left\{\mathbf{x} \in \mathbb{R}^{d} \mid \operatorname{dist}(x, D)<r\right\}$. In what follows, when we refer to an $\mathrm{O}(\varepsilon)$ neighbourhood of a set, we mean an $\varepsilon$-dependent system of $r$-neighbourhoods in which, for $\varepsilon$ sufficiently small, $r=c \varepsilon$ for some unspecified constant $c$.

In order to understand the set-theoretic limit, we will investigate the behaviour of the images $\mathbf{f}_{\varepsilon}(A)$ of certain subsets $A \subset \mathbb{S}^{d-1}$, gradually building up to the entire sphere. Let us begin with the simplest case: the images of a curve $C \subset \mathbb{S}^{d-1}$. If $C \subset S_{i}^{\circ}$ is entirely contained in the interior of the normal spherical polytope associated with a vertex $\mathbf{x}_{i}$ for some $1 \leq i \leq \kappa$, then by equation (7.1), $\mathbf{f}_{\mathcal{E}}(C) \rightarrow\left\{\mathbf{x}_{i}\right\}$ as $\varepsilon \rightarrow 0^{+}$.

The next simplest case is when the curve $C$ is contained in the union of two adjacent vertex spherical polytopes. Thus, by relabelling, let $\mathbf{x}_{1}, \mathbf{x}_{2}$ be adjacent vertices of $B$. Let

$$
E=\left\{\lambda_{1} \mathbf{x}_{1}+\lambda_{2} \mathbf{x}_{2} \mid \lambda_{1}, \lambda_{2} \geq 0, \lambda_{1}+\lambda_{2}=1\right\} \subset B
$$

denote the edge connecting $\mathbf{x}_{1}$ to $\mathbf{x}_{2}$. Suppose that its interior contains $l \geq 0$ additional points in the configuration, which we number as $\mathbf{x}_{3}, \ldots, \mathbf{x}_{l+2} \in E^{\circ}$, while the remaining points $\mathbf{x}_{l+3}, \ldots, \mathbf{x}_{n} \in K \backslash E$. We note that we can also write, redundantly,

$$
E=\left\{\sum_{i=1}^{l+2} \lambda_{i} \mathbf{x}_{i} \mid \lambda_{i} \geq 0, \sum_{i=1}^{l+2} \lambda_{i}=1\right\}
$$

Let $S_{1}, S_{2} \subset \mathbb{S}^{d-1}$ be the normal spherical polytopes associated with $\mathbf{x}_{1}, \mathbf{x}_{2}$, respectively, while $S_{E}=\partial S_{1} \cap \partial S_{2}$ is the normal spherical polytope associated with the edge $E$. Thus $S_{1}^{\circ}, S_{2}^{\circ}$ are open subsets of $\mathbb{S}^{d-1}$, while $S_{E}^{\circ}$ is a $(d-2)$-dimensional submanifold. Consider a curve $C \subset S_{1}^{\circ} \cup S_{2}^{\circ} \cup S_{E}^{\circ} \subset \mathbb{S}^{d-1}$ such that one endpoint of $C$ lies in $S_{1}^{\circ}$ and the other lies in $S_{2}^{\circ}$, which, by connectivity, implies $C \cap S_{E}^{\circ} \neq \varnothing$. Our goal is to prove that the image curves $\mathbf{f}_{\varepsilon}(C)$ converge, as sets, to the edge $E$.

Now, if $\mathbf{n} \in C \cap S_{1}^{\circ}$, Lemma 6.1 combined with equations (2.4) and (6.4) implies

$$
\lambda_{1}(\varepsilon, \mathbf{n})=1+\varepsilon q_{1}(\varepsilon, \mathbf{n}), \quad \lambda_{j}(\varepsilon, \mathbf{n})=\varepsilon q_{j}(\varepsilon, \mathbf{n}), \quad j=2,3, \ldots, n,
$$

where $q_{1}, \ldots, q_{n}$ are rational functions of $\varepsilon$ depending continuously on $\mathbf{n} \in C$. Thus, formula (7.3) reestablishes the fact that all of the points in $\mathbf{f}_{\varepsilon}\left(C \cap S_{1}^{\circ}\right)$ converge to the vertex $\mathbf{x}_{1}$ as $\varepsilon \rightarrow 0^{+}$. A similar statement holds for $\mathbf{n} \in C \cap S_{2}^{\circ}$ :

$$
\lambda_{2}(\varepsilon, \mathbf{n})=1+\varepsilon q_{2}(\varepsilon, \mathbf{n}), \quad \lambda_{j}(\varepsilon, \mathbf{n})=\varepsilon q_{j}(\varepsilon, \mathbf{n}), \quad j=1,3, \ldots, n .
$$


Finally, if $\mathbf{n} \in C \cap S_{E}^{\circ}$, in view of equations (2.4), (2.5) and (6.5), we have

$$
\lambda_{i}(\varepsilon, \mathbf{n})= \begin{cases}\frac{d_{i}(\mathbf{n})+\varepsilon p_{i}(\varepsilon, \mathbf{n})}{D(\mathbf{n})+\varepsilon P(\varepsilon, \mathbf{n})}, & i=1, \ldots, l+2, \\ \frac{\varepsilon p_{i}(\varepsilon, \mathbf{n})}{D(\mathbf{n})+\varepsilon P(\varepsilon, \mathbf{n})}, & i=l+3, \ldots, n,\end{cases}
$$

where

$$
D(\mathbf{n})=\sum_{i=1}^{l+2} d_{i}(\mathbf{n})>0, \quad P(\varepsilon, \mathbf{n})=\sum_{i=1}^{n} p_{i}(\varepsilon, \mathbf{n})
$$

Comparing with equations (7.3), (7.4) and (7.5), we find that for any $\mathbf{n} \in C$,

$$
\sum_{i=1}^{l+2} \lambda_{i}(\varepsilon, \mathbf{n})=1+\varepsilon Q(\varepsilon, \mathbf{n}), \quad \mathbf{f}_{\varepsilon}(\mathbf{n})=\sum_{i=1}^{l+2} \lambda_{i}(\varepsilon, \mathbf{n}) \mathbf{x}_{i}+\varepsilon R(\varepsilon, \mathbf{n})
$$

where both $Q$ and $R$ are continuous functions of $\mathbf{n} \in C$, including when $\mathbf{n} \in S_{E}^{\circ}$, and rational functions of $\varepsilon$ with nonvanishing denominator. Since $C \subset \mathbb{S}^{d-1}$ is compact, they can thus be bounded by an overall constant independent of $\varepsilon \in\left(0, \varepsilon_{0}\right]$. This holds even at the singular point $\mathbf{n} \in C \cap S_{E}^{\circ}$ when there is cancellation of powers of $\varepsilon$ in numerator and denominator, whence equation (7.5). Thus, comparing with equation (7.2), we deduce that for $0<\varepsilon \leq \varepsilon_{0}$, there exists an $\mathrm{O}(\varepsilon)$ neighbourhood $U_{\varepsilon}$ of the edge $E$ such that the images $\mathbf{f}_{\mathcal{E}}(C) \subset U_{\mathcal{E}}$. This immediately implies that the limiting set is contained within the edge: $\lim _{\varepsilon \rightarrow 0^{+}} \mathbf{f}_{\varepsilon}(C) \subset E$. The remaining task is to prove that every point in $E$ is contained in the limit, and therefore $\lim _{\varepsilon \rightarrow 0^{+}} \mathbf{f}_{\varepsilon}(C)=E$.

We already know that both endpoints $\mathbf{x}_{1}, \mathbf{x}_{2}$ are contained in the limiting set. Thus, given a point $\mathbf{x} \in E^{\circ}$, we need to find points $\mathbf{y}_{\varepsilon} \in \mathbf{f}_{\varepsilon}(C)$ that converge to $\mathbf{x}=\lim _{\varepsilon \rightarrow 0^{+}} \mathbf{y}_{\mathcal{\varepsilon}}$. Although it is possible to do this by a careful analysis of the underlying formulae, we prefer, for later purposes, to use a simple topological proof.

To this end, let $Z_{\mathbf{x}}$ be the affine hyperplane passing through $\mathbf{x}$ that is orthogonal to $E$, and define $Z_{\mathbf{x}, \varepsilon}=Z_{\mathbf{x}} \cap U_{\mathcal{\varepsilon}}$. We claim that there exists $\mathbf{y}_{\varepsilon} \in \mathbf{f}_{\varepsilon}(C) \cap Z_{\mathbf{x}, \varepsilon}$. If true, then we have produced the desired points. To prove the claim, observe that $U_{\varepsilon} \backslash Z_{\mathbf{x}, \varepsilon}$ consists of two disjoint open subsets, say $U_{\mathbf{x}, \varepsilon}^{1}, U_{\mathbf{x}, \varepsilon}^{2}$, with $\mathbf{x}_{i} \in U_{\mathbf{x}, \varepsilon}^{i}$ for $i=1,2$. Moreover, since we know that all the points in $\mathbf{f}_{\varepsilon}\left(C \cap S_{i}^{\circ}\right)$ converge to $\mathbf{x}_{i}$, if we choose $\varepsilon$ sufficiently small, then $\mathbf{f}_{\varepsilon}(C) \cap U_{\mathbf{x}, \varepsilon}^{i} \neq \varnothing$. Therefore, $\mathbf{f}_{\varepsilon}(C) \cap Z_{\mathbf{x}, \varepsilon}=\varnothing$ would contradict the connectedness of $\mathbf{f}_{\varepsilon}(C)$. This contradiction establishes our claim. We thus conclude that, as sets,

$$
\mathbf{f}_{\varepsilon}(C) \longrightarrow E \quad \text { as } \quad \varepsilon \longrightarrow 0
$$

For later purposes, we need slightly more than mere set-theoretic convergence (7.7). Namely, we require the existence of a continuous set-valued homotopy that connects the images of $\mathbf{f}_{\varepsilon}: C \rightarrow K$ for $\varepsilon>0$ to a set-valued map $\widehat{\mathbf{f}}_{0}: C \rightarrow 2^{K}$ with range equal to the edge $E=\widehat{\mathbf{f}}_{0}(C)$, a model being Example 4.1. Rather than write down an explicit formula for this homotopy, we will instead construct its graph.

Consider the graph

$$
\Gamma=\left\{\left(\varepsilon, \mathbf{n}, \mathbf{f}_{\varepsilon}(\mathbf{n})\right) \mid 0<\varepsilon \leq \varepsilon_{0}, \mathbf{n} \in C\right\} \subset\left(0, \varepsilon_{0}\right] \times C \times K
$$

of the map $F(\varepsilon, \mathbf{n})=\mathbf{f}_{\varepsilon}(\mathbf{n})$ for $0<\varepsilon \leq \varepsilon_{0}$ and $\mathbf{n} \in C$. Let $\bar{\Gamma}=$ Clos $\Gamma$ be its closure in $\left[0, \varepsilon_{0}\right] \times C \times K$. 
According to the preceding proof, $\bar{\Gamma}$ is the graph of the set-valued map $F:\left[0, \varepsilon_{0}\right] \times C \rightarrow 2^{K}$ given by

$$
F(\varepsilon, \mathbf{n})=\left\{\begin{array}{lll}
\mathbf{f}_{\varepsilon}(\mathbf{n}), & \varepsilon>0, \\
\mathbf{x}_{1}, & \varepsilon=0, & \mathbf{n} \in C \cap S_{1}^{\circ}, \\
\mathbf{x}_{2}, & \varepsilon=0, & \mathbf{n} \in C \cap S_{2}^{\circ}, \\
E, & \varepsilon=0, & \mathbf{n} \in C \cap S_{E}^{\circ},
\end{array}\right.
$$

its final value being the entire edge $E \subset K$. Moreover, since $\bar{\Gamma}$ is closed and $K$ is compact and Hausdorff, the closed graph theorem for set-valued functions [2, Prop. 1.4.8] implies that the set-valued function $F$ is upper hemicontinuous, as per Definition 4.2. Thus for all $0<\varepsilon \leq \varepsilon_{0}$, equation (7.8) defines an upper hemicontinuous homotopy from each $\mathbf{f}_{\varepsilon}: C \rightarrow K$ to the set-valued map $\widehat{\mathbf{f}}_{0}: C \rightarrow 2^{K}$ with $\widehat{\mathbf{f}}_{0}(\mathbf{n})=F(0, \mathbf{n})$, whose range $\widehat{\mathbf{f}}_{0}(C)$ is the edge $E$.

Remark. An alternative approach, that avoids set-valued homotopies and, later, set-valued homology, is to 'tilt' the subset $\bar{\Gamma}$ so that it becomes a graph by introducing new coordinates on the Cartesian product space $\left[0, \varepsilon_{0}\right] \times C \times K$. However, this is more technically tricky to accomplish in the higher-dimensional cases to be handled later, and the set-theoretic approach provides a cleaner path to the proof.

The remainder of the proof works by induction on the dimension of the face $F$. Thus the next case is that of a two-dimensional face $F \subset B \subset \mathbb{R}^{d}$. The main steps of the proof in this situation will then be straightforwardly adapted to any higher-dimensional face. Let $\mathbf{x}_{1}, \ldots, \mathbf{x}_{k}$ be the vertices of $F$ and let $E_{1}, \ldots, E_{k}$ be its edges. We label the vertices and edges so that $E_{j}$ connects $\mathbf{x}_{j}$ to $\mathbf{x}_{j+1}$, with indices taken modulo $k$ throughout, whence $\mathbf{x}_{k+1}=\mathbf{x}_{1}$. Thus $E=\bigcup_{j=1}^{k} E_{j}=\partial F$ is the polygonal boundary of $F$. We assume that there are $l \geq 0$ additional points $\mathbf{x}_{k+1}, \ldots, \mathbf{x}_{k+l} \in F \backslash\left\{\mathbf{x}_{1}, \ldots, \mathbf{x}_{k}\right\}$, with the remaining points in the configuration $\mathbf{x}_{k+l+1}, \ldots, \mathbf{x}_{n} \in K \backslash F$. Keep in mind that $F$ is convex.

Let $S_{i}, \widetilde{S}_{j}, S_{F}$ be the normal spherical polytopes of $\mathbf{x}_{i}, E_{j}, F$, respectively, so that $\widetilde{S}_{j} \subset \partial S_{j} \cap \partial S_{j+1}$ and $S_{F} \subset \partial \widetilde{S}_{j}$ for all $j=1, \ldots, k$. Let $W_{F} \subset \mathbb{S}^{d-1}$ be the open set given by equation (3.13) or (3.14), and let $\widetilde{N} \subset N \subset W_{F}$ be the two-dimensional submanifolds satisfying the hypotheses of Proposition 3.4. As in equation (7.6), applying Lemma 6.2 we find

$$
\sum_{i=1}^{k+l} \lambda_{i}(\varepsilon, \mathbf{n})=1+\varepsilon Q(\varepsilon, \mathbf{n}), \quad \mathbf{f}_{\varepsilon}(\mathbf{n})=\sum_{i=1}^{k+l} \lambda_{i}(\varepsilon, \mathbf{n}) \mathbf{x}_{i}+\varepsilon R(\varepsilon, \mathbf{n}),
$$

where both $Q$ and $R$ are continuous functions of $\mathbf{n} \in N$, and rational functions of $\varepsilon$ with nonvanishing denominator. These formulae again imply that the images $\mathbf{f}_{\varepsilon}(N)$ lie in an $\mathrm{O}(\varepsilon)$ neighbourhood $U_{\varepsilon}$ of the face $F$, and hence $\lim _{\varepsilon \rightarrow 0^{+}} \mathbf{f}_{\varepsilon}(N) \subset F$. The remaining task is to prove that every point in $F$ is contained in the limit, a result that requires a more sophisticated topological argument than in the curve case.

For this purpose, we replace $N$ by $\widetilde{N}$. Clearly, if we can prove $\lim _{\varepsilon \rightarrow 0^{+}} \mathbf{f}_{\varepsilon}(\widetilde{N})=F$, by the preceding result the same is true of $N \supset \widetilde{N}$. According to Proposition 3.4, $\widetilde{N} \cap S_{j}^{\circ} \neq \varnothing$ and $\widetilde{N} \cap \widetilde{S}_{j}^{\circ} \neq \varnothing$ for all $j=1, \ldots, k$, and either $\mathbf{n}_{F} \in \widetilde{N}$ when $d=3$, where $\mathbf{n}_{F}$ is the unit outward normal to the polyhedral facet $F$, or $\widetilde{N} \cap S_{F}^{\circ} \neq \varnothing$ when $d>3$. Moreover, the boundary $L=\partial \widetilde{N}$ can be decomposed into curves $L_{1}, \ldots, L_{k}$, any two of which overlap only on their common boundaries, that satisfy $L_{j} \subset S_{j}^{\circ} \cup \widetilde{S}_{j}^{\circ} \cup S_{j+1}^{\circ}$, again modulo $k$. Let $\left\{\mathbf{n}_{j}\right\}=L_{j-1} \cap L_{j} \subset S_{j}^{\circ}$ denote the common endpoints of adjacent curves in $L=\partial \widetilde{N}$.

Let us set $I=\left[0, \varepsilon_{0}\right]$ for $\varepsilon_{0}>0$ sufficiently small. According to the preceding curve proof, $\mathbf{f}_{\varepsilon}\left(L_{j}\right) \rightarrow E_{j}$ as sets, and moreover, there exists an upper hemicontinuous homotopy (of set-valued mappings) from each $\mathbf{f}_{\varepsilon}: L_{j} \rightarrow K$ for all $0<\varepsilon \leq \varepsilon_{0}$ to the set-valued limit $\widehat{\mathbf{f}}_{0}: L_{j} \rightarrow 2^{K}$ with range 
equal to the edge $E_{j}=\widehat{\mathbf{f}}_{0}\left(L_{j}\right)$. The graph of this homotopy,

$$
\Gamma_{j} \subset I \times L_{j} \times K \subset I \times \mathbb{S}^{2} \times K,
$$

is a closed subset of the indicated Cartesian product space.

We now piece together these homotopy graphs to define a homotopy from $\mathbf{f}_{\mathcal{E}}(L)$ to $E=\partial F$ whose graph is

$$
\Gamma=\bigcup_{j=1}^{k} \Gamma_{j} \subset I \times L \times K \subset I \times \mathbb{S}^{2} \times K .
$$

Note that $\Gamma$ is a closed subset that defines the graph of an upper hemicontinuous function, because each $\Gamma_{j}$ is closed, and moreover,

$$
\Gamma_{j-1} \cap\left(I \times\left\{\mathbf{n}_{j}\right\} \times K\right)=\Gamma_{j} \cap\left(I \times\left\{\mathbf{n}_{j}\right\} \times K\right),
$$

including when $\varepsilon=0$, since $\mathbf{f}_{\varepsilon}\left(\mathbf{n}_{j}\right) \rightarrow \mathbf{x}_{j}$, and so

$$
\Gamma_{j-1} \cap\left(\{0\} \times\left\{\mathbf{n}_{j}\right\} \times K\right)=\left\{\left(0, \mathbf{n}_{j}, \mathbf{x}_{j}\right)\right\}=\Gamma_{j} \cap\left(\{0\} \times\left\{\mathbf{n}_{j}\right\} \times K\right) .
$$

Given $\mathbf{x} \in F^{\circ}$, we seek $\mathbf{y}_{\varepsilon} \in \mathbf{f}_{\varepsilon}(\widetilde{N})$ that converge to $\mathbf{x}$ as $\varepsilon \rightarrow 0^{+}$. Let $Z_{\mathbf{x}}$ be the affine subspace of dimension $d-2$ passing through $\mathbf{x}$ that is orthogonal to $F$. Define $Z_{\mathbf{x}, \varepsilon}=Z_{\mathbf{x}} \cap U_{\varepsilon}$. Again, if we can prove that there exists $\mathbf{y}_{\varepsilon} \in Z_{\mathbf{x}, \varepsilon} \cap \mathbf{f}_{\varepsilon}(\widetilde{N})$, we are done. Suppose not - that is, suppose that $\mathbf{f}_{\varepsilon}(\widetilde{N}) \subset U_{\varepsilon} \backslash Z_{\mathbf{x}, \varepsilon}$. The idea is to demonstrate that this is topologically impossible due to the contractibility of $\widetilde{N}$, and hence of $\mathbf{f}_{\varepsilon}(\widetilde{N})$, whereas $\mathbf{f}_{\varepsilon}(L)=\mathbf{f}_{\varepsilon}(\partial \widetilde{N})$, for $\varepsilon$ sufficiently small, defines a nontrivial homology class in $U_{\varepsilon} \backslash Z_{\mathbf{x}, \varepsilon}$.

If we were dealing with ordinary mappings, this topological argument would be straightforward. But because $\widehat{\mathbf{f}}_{0}$ is a set-valued mapping, we will need some more sophisticated tools from set-valued algebraic topology to establish the contradiction. We summarise the basic theory, based on a paper of $\mathrm{Li}$ [11], in Appendix A. In accordance with the notation introduced there, we use italic $H_{n}(X)$ to denote the standard $n$ th-order singular homology groups of a topological space $X$ and calligraphic $\mathcal{H}_{n}(X, \mathcal{U})$ to denote the corresponding $n$ th-order set-valued homology groups relative to a chosen open cover $\mathcal{U}$. (As noted in Appendix A, if this cover is not chosen carefully, the set-valued homology groups are all trivial, and would hence be useless for the present purposes.)

In this situation, we select the particular open covering $\mathcal{V}$ of $U_{\varepsilon} \backslash Z_{\mathbf{x} ; \varepsilon}$ consisting of all open sets of the form

$$
V=H \cap\left(U_{\varepsilon} \backslash Z_{\mathbf{x} ; \varepsilon}\right) \quad \text { such that } \quad \mathbf{x} \in \partial V
$$

where $H$ is an open half space in $\mathbb{R}^{d}$. We claim that $\mathcal{V}$ satisfies Li's contractible finite intersection property, because the intersection of any finite collection of such open sets, if nonempty, is homeomorphic to the Cartesian product of an open $(d-2)$-dimensional ball with an open circular sector, meaning the intersection of an acute-angled open planar cone with the unit disk (a pizza slice), which is clearly contractible.

The limiting set-valued function $\widehat{\mathbf{f}}_{0}: L \rightarrow 2^{E}$, whose range is the polygonal boundary of the face $\widehat{\mathbf{f}}_{0}(L)=E=\partial F$, is compatible with the open covering $\mathcal{V}$, because $\widehat{\mathbf{f}}_{0}(\mathbf{n})$ is either a vertex or an edge $E_{j}$. Moreover, when $\varepsilon>0$, the map $\mathbf{f}_{\varepsilon}$ is continuous and single-valued, which implies trivially that its restriction to $L$ is compatible with any open covering of $U_{\varepsilon} \backslash Z_{\mathbf{x} ; \varepsilon}$.

The family of maps $\left\{\mathbf{f}_{\varepsilon}, \widehat{\mathbf{f}}_{0}\right\}$ thus defines, by varying $\varepsilon$, an upper hemicontinuous homotopy of multivalued functions with closed values. It follows from [11, Prop. 6] that the upper triangle in Figure 5 commutes. The square is divided into two triangles. It follows from the definitions that the top right triangle in the square commutes. As noted in Appendix A, the map $i_{\sharp}$ on the bottom right is an 


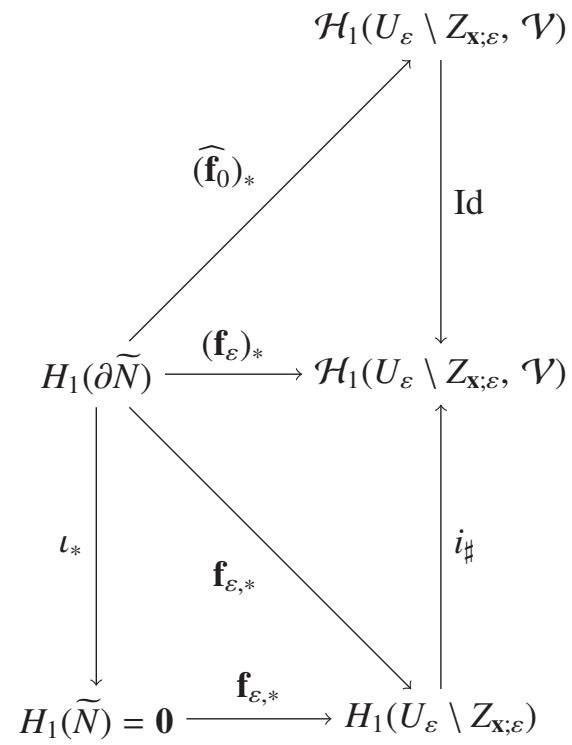

Figure 5. Commutative diagram

isomorphism. Finally, the map $\iota: L=\partial \widetilde{N} \rightarrow \widetilde{N}$ denotes the inclusion map, and so it is a standard fact from ordinary singular homology theory that the bottom left triangle commutes.

Let $0 \neq c=[\partial \widetilde{N}] \in H_{1}(\partial \widetilde{N})$ be the homology class representing $\partial \widetilde{N}$, which is in fact a generator. We claim ${ }^{4}$ that $\left(\widehat{\mathbf{f}}_{0}\right)_{*}(c)$ is a nonzero element of $H_{1}\left(U_{\varepsilon} \backslash Z_{\mathbf{x} ; \varepsilon}, \mathcal{V}\right)$. Indeed, $\left(\widehat{\mathbf{f}}_{0}\right)_{*}(c)=i_{\sharp}([E]) \neq 0$, since the homology class $[E]=[\partial F] \in H_{1}\left(U_{\varepsilon} \backslash Z_{\mathbf{x} ; \varepsilon}\right)$ is nonzero and $i_{\sharp}$ is an isomorphism. This thus proves the claim that

$$
\left(\mathbf{f}_{\varepsilon}\right)_{*}(c)=\left(\widehat{\mathbf{f}}_{0}\right)_{*}(c) \neq 0, \quad \text { and hence } \quad \mathbf{f}_{\varepsilon, *}(c)=i_{\sharp}^{-1}\left[\left(\mathbf{f}_{\varepsilon}\right)_{*}(c)\right] \neq 0 .
$$

On the other hand, the bottom left triangle in the square in Figure 5 shows that $\mathbf{f}_{\varepsilon, *}$ vanishes identically on $H_{1}(\partial \widetilde{N})$, so that $\mathbf{f}_{\varepsilon, *}(c)=0$, thus leading to the desired contradiction and establishing the existence of $\mathbf{y}_{\varepsilon} \in \mathbf{f}_{\varepsilon}(\widetilde{N})$. This finishes the proof that every point of $F$ belongs to the inner limit of $\mathbf{f}_{\varepsilon}(\widetilde{N})$ as $\varepsilon \rightarrow 0$. We conclude that both $\mathbf{f}_{\mathcal{E}}(\widetilde{N})$ and $\mathbf{f}_{\mathcal{\varepsilon}}(N) \rightarrow F$ as sets as $\varepsilon \rightarrow 0^{+}$.

Finally, to establish the existence of a set-valued homotopy connecting the maps $\mathbf{f}_{\varepsilon}: N \rightarrow K$ to the set-valued map $\widehat{\mathbf{f}}_{0}: N \rightarrow 2^{K}$ with range $F=\widehat{\mathbf{f}}_{0}(N)$, we proceed as follows. As in the curve case, we construct its graph $\bar{\Gamma} \subset I \times N \times K$ as the closure of the graph

$$
\Gamma=\left\{\left(\varepsilon, \mathbf{n}, \mathbf{f}_{\varepsilon}(\mathbf{n}) \mid \mathbf{n} \in N, 0<\varepsilon \leq \varepsilon_{0}\right\} \subset\left(0, \varepsilon_{0}\right] \times N \times K\right.
$$

of the continuous map $F(\varepsilon, \mathbf{n})=\mathbf{f}_{\varepsilon}(\mathbf{n})$. Again, by the closed graph theorem for set-valued functions, $\bar{\Gamma}$ is the graph of an upper hemicontinuous set-valued function $F: I \times N \rightarrow 2^{K}$ which, for $0<\varepsilon \leq \varepsilon_{0}$, defines the required upper hemicontinuous homotopy.

Finally, let us outline the proof in the general case. To this end, we establish the following result by induction on the dimension $m$ of the face, using the preceding two-dimensional case as a model:

Proposition 7.1. Let $F \subset B$ be an m-dimensional face, and let $N \subset \mathbb{S}^{d-1}$ be an $m$-dimensional submanifold satisfying the conditions of Proposition 3.4. Then the set-theoretic $\lim _{\varepsilon \rightarrow 0^{+}} \mathbf{f}_{\mathcal{\varepsilon}}(N)=F$. Moreover, for $\varepsilon>0$ sufficiently small, there is a continuous set-valued homotopy from $\mathbf{f}_{\varepsilon}: N \rightarrow K$ to the set-valued map $\widehat{\mathbf{f}}_{0}: N \rightarrow 2^{K}$ whose range is the entire face: $\mathbf{f}_{0}(N)=F$.

${ }^{4}$ As in Appendix A, the parentheses indicate the induced maps on set-theoretic homology. 
Referring back to the preceding argument for two-dimensional polygonal faces, the key formulae (7.9) work exactly as before, with $\mathbf{x}_{1}, \ldots, \mathbf{x}_{k}$ the vertices of $F$ and $\mathbf{x}_{k+1}, \ldots, \mathbf{x}_{k+l}$ additional points in the configuration, if any, in $F \backslash\left\{\mathbf{x}_{1}, \ldots, \mathbf{x}_{k}\right\}$. These in turn imply that, for $\varepsilon$ sufficiently small, the images $\mathbf{f}_{\varepsilon}(N)$ lie in an $\mathrm{O}(\varepsilon)$ neighbourhood of $F$, thus proving that $\lim _{\varepsilon \rightarrow 0^{+}} \mathbf{f}_{\varepsilon}(N) \subset F$.

To prove that every point in $\mathbf{x} \in F$ is contained in the limit, we replace $N$ by the open submanifold $\widetilde{N} \subset$ $N$ given in Proposition 3.4. Again assume the contrary, that $\mathbf{f}_{\varepsilon}(\widetilde{N}) \subset U_{\varepsilon} \backslash Z_{\mathbf{x}, \varepsilon}$, where $Z_{\mathbf{x}, \varepsilon}=Z_{\mathbf{x}} \cap U_{\varepsilon}$ with $Z_{\mathbf{x}}$ the affine subspace of dimension $d-m$ passing through $\mathbf{x}$ orthogonal to $F$. According to the inductive hypothesis, its $(m-1)$-dimensional boundary component $L_{i} \subset \partial \widetilde{N}$ satisfies $\lim _{\mathcal{\varepsilon} \rightarrow 0^{+}} \mathbf{f}_{\mathcal{\varepsilon}}\left(L_{i}\right)=$ $G_{i}$, the corresponding $(m-1)$-dimensional subface of $F$, through an upper hemicontinuous homotopy from $\mathbf{f}_{\varepsilon}: L_{i} \rightarrow K$ to the set-valued map $\widehat{\mathbf{f}}_{0}: L_{i} \rightarrow 2^{K}$ with range $G_{i}=\widehat{\mathbf{f}}_{0}\left(L_{i}\right)$. We then, as in equation (7.10), piece together these subface homotopies so as to construct an upper hemicontinuous homotopy from $\mathbf{f}_{\varepsilon}: \partial \widetilde{N} \rightarrow K$ to the set-valued map $\widehat{\mathbf{f}}_{0}: \partial \widetilde{N} \rightarrow 2^{K}$ whose range is all of $\partial F=\widehat{\mathbf{f}}_{0}(\partial \widetilde{N})$.

The topological argument then proceeds in an identical manner, the only difference being that the open cover $\mathcal{V}$ is constructed as in equation (7.11), but now the intersections are homeomorphic to the contractible Cartesian product of a spherical sector of dimension $m$ with a ball of dimension $d-m$. Further, we use the same commutative diagram as in Figure 5 but with the first homology group $\mathcal{H}_{1}$ replaced by $\mathcal{H}_{m-1}$ throughout. The resulting topological contradiction proves that

$$
\lim _{\varepsilon \rightarrow 0^{+}} \mathbf{f}_{\varepsilon}(\widetilde{N})=\lim _{\varepsilon \rightarrow 0^{+}} \mathbf{f}_{\varepsilon}(N)=F .
$$

Finally, the construction of the corresponding upper hemicontinuous set-valued homotopy from $\mathbf{f}_{\varepsilon}: N \rightarrow K$ to $\widehat{\mathbf{f}}_{0}: N \rightarrow 2^{K}$ with range $\widehat{\mathbf{f}}_{0}(N)=F$ proceeds exactly as before.

The final step in the proof of Theorem 2.1 is to prove that $\lim _{\varepsilon \rightarrow 0^{+}} \mathbf{f}_{\varepsilon}\left(\mathbb{S}^{d-1}\right)=B$. For this, we split up $B$ into its facets $B=F_{1} \cup \cdots \cup F_{k}$. For each $F_{i}$, by combining Lemma 6.2 with the argument following equation (7.9), we deduce that $\lim _{\varepsilon \rightarrow 0^{+}} \mathbf{f}_{\varepsilon}\left(S_{F_{i}}\right) \subset F_{i}$, and hence $\lim _{\varepsilon \rightarrow 0^{+}} \mathbf{f}_{\varepsilon}\left(\mathbb{S}^{d-1}\right) \subset B$. On the other hand, by the case $m=d-1$ of Proposition 7.1, there exists a $(d-1)$-dimensional submanifold $N_{i} \subset \mathbb{S}^{d-1}$ such that $\lim _{\varepsilon \rightarrow 0^{+}} \mathbf{f}_{\varepsilon}\left(N_{i}\right)=F_{i}$. We conclude that $\lim _{\varepsilon \rightarrow 0^{+}} \mathbf{f}_{\varepsilon}\left(\mathbb{S}^{d-1}\right)=B$, as desired. Moreover, we can similarly piece together the set-valued homotopies for each facet to find a set-valued homotopy from $\mathbf{f}_{\varepsilon}: \mathbb{S}^{d-1} \rightarrow K$ to the set-valued inverse Gauss map (5.2) mapping the sphere to the boundary polytope $B=\partial K$. This, at last, completes the proof.

\section{Appendix A. Set-valued homology}

In this appendix, we review the basics of set-valued singular homology following Li [11]. For simplicity, we will use $\mathbb{Q}$ as the ring of coefficients throughout.

Let $X, Y$ be connected normal Hausdorff topological spaces. Given a set-valued mapping $F: X \rightarrow 2^{Y}$ and an open covering $\mathcal{U}$ of $Y$, we say that $F$ is compatible with $\mathcal{U}$ if and only if for any $x \in X$ there is some $U \in \mathcal{U}$ such that $F(x) \subset U$. Define

$$
\mathcal{C}(X, Y, \mathcal{U})=\left\{\begin{array}{l|l}
F: X \rightarrow 2^{Y} & \begin{array}{l}
F \text { is an upper hemicontinuous mapping } \\
\text { with closed values compatible with } \mathcal{U}
\end{array}
\end{array}\right\}
$$

Let

$$
\Delta_{n}=\left\{x=\left(x_{0}, \ldots, x_{n}\right) \in \mathbb{R}^{n+1} \mid x_{i} \geq 0, x_{0}+x_{1}+\cdots+x_{n}=1\right\}
$$

denote the standard $n$-dimensional simplex. For $i=0, \ldots, n$, let

$$
\varphi_{i}\left(x_{0}, \ldots, x_{n}\right)=\left(x_{0}, \ldots, x_{i-1}, 0, x_{i}, \ldots, x_{n}\right)
$$

map the $(n-1)$-dimensional simplex $\Delta_{n-1}$ to the $i$ th face $\Delta_{n}^{(i)}=\Delta_{n} \cap\left\{x_{i}=0\right\}$ of the $n$-dimensional simplex. 
Given an open cover $\mathcal{U}$ of $Y$, we define the $n$th set-valued chain group $C_{n}(Y, \mathcal{U})$ to be the free abelian group generated by $C\left(\Delta_{n}, Y, \mathcal{U}\right)$. We then define the boundary operator $\partial_{n}: C_{n}(Y, \mathcal{U}) \rightarrow C_{n-1}(Y, \mathcal{U})$ by

$$
\partial_{n} c_{n}=\sum_{i=0}^{n}(-1)^{i} c_{n} \circ \varphi_{i}
$$

Thus, $\partial_{n} \circ \partial_{n+1}=0$, which is usually abbreviated by $\partial^{2}=0$.

The $n$th set-valued homology group of $(Y, \mathcal{U})$ is then given by

$$
\mathcal{H}_{n}(Y, \mathcal{U})=\operatorname{Ker} \partial_{n} / \operatorname{Im} \partial_{n+1} .
$$

As noted by $\mathrm{Li}$ [11], if one is not careful when choosing the cover $\mathcal{U}$, all set-valued homology groups are trivial, and would thus be of no help establishing the desired topological result. To avoid this difficulty, $\mathrm{Li}$ imposes the contractible finite intersection property on the cover $\mathcal{U}$. This property requires that the intersection of any finite collection of elements of the cover be either empty or contractible.

Since ordinary functions can be viewed as set-valued functions, there is a natural inclusion map $i$ from the $n$th chain group $C_{n}(Y)$, as defined in the usual singular homology theory, to $C_{n}(Y, \mathcal{U})$. The inclusion is a chain map, and thus induces a group homomorphism

$$
i_{\sharp}: H_{n}(Y) \longrightarrow \mathcal{H}_{n}(Y, \mathcal{U}),
$$

which according to [11, Theorem 11] is actually an isomorphism.

Moreover, an upper hemicontinuous set-valued mapping $F: X \rightarrow 2^{Y}$ with closed values induces a chain map from $C_{n}(X)$ to $C_{n}(Y, \mathcal{U})$, and thus induces a group homomorphism

$$
(F)_{*}: H_{n}(X) \longrightarrow \mathcal{H}_{n}(Y, \mathcal{U}) .
$$

In general, we will place parentheses around $(F)_{*}$ in order to distinguish the set-valued homology group homomorphism from the usual group homomorphism $f_{*}: H_{n}(X) \rightarrow H_{n}(Y)$ on the corresponding singular homology groups induced by a continuous (ordinary) function $f: X \rightarrow Y$. Further results of $\mathrm{Li}[11]$ are quoted in the text as needed.

Acknowledgements. We thank the anonymous referee for corrections, suggestions and references, and in particular for thoughtprovoking remarks on our proof using the point of view of convergence with respect to the Hausdorff metric. We also would like to thank Elias Saleeby, Dennis Sullivan, Daniele Tampieri, Paolo Emilio Ricci and Kamal Khuri-Makdisi for their useful remarks and suggestions. The figures were mostly generated using Python 3, though the polyhedra in Figure 4 were generated using ParaView.

Conflict of Interest: None.

\section{References}

[1] A. V. Arutyunov and V. Obukhovskii, Convex and Set-Valued Analysis: Selected Topics (De Gruyter, Berlin, 2017).

[2] J. P. Aubin and H. Frankowska, Set-Valued Analysis (Birkhäuser, Boston, 2009).

[3] D. Avis, D. Bremner and R. Seidel, 'How good are convex hull algorithms?', Comput. Geom. 7 (1997), $265-301$.

[4] S. P. Boyd and L. Vandenberghe, Convex Optimization (Cambridge University Press, Cambridge, UK, 2004).

[5] H. Chen and A. Padrol, 'Scribability problems for polytopes', European J. Combin. 64 (2017), 1-26.

[6] W. J. Firey, 'Approximating convex bodies by algebraic ones', Arch. Math. (Basel) 25 (1974), 424-425.

[7] M. Ghomi, 'Optimal smoothing for convex polytopes', Bull. Lond. Math. Soc. 36 (2004), 483-492.

[8] A. Gray, E. Abbena and S. Salamon, Modern Differential Geometry of Curves and Surfaces with Mathematica, third edn (Chapman \& Hall/CRC, Boca Raton, FL, 2006).

[9] B. Grünbaum, Convex Polytopes, second edn, Graduate Texts in Mathematics, 227 (Springer-Verlag, New York, 2003).

[10] C. Kuratowski, Topologie I (Monografje Matematyczne, Warszawa-Lwow, 1933).

[11] Y. Li, 'Set-valued homology', Topology Appl. 83 (1998) 149-158.

[12] H. Maehara and H. Martini, 'Outer normal transforms of convex polytopes', Results Math. 72 (2017), 87-103. 
[13] P. McMullen and G. C. Shephard, Convex Polytopes and the Upper Bound Conjecture, London Math. Soc. Lecture Note Series, 3 (Cambridge University Press, Cambridge, UK, 1971).

[14] H. Minkowski 'Volumen und Oberfläche', Math. Ann. 57 (1903), 447-495.

[15] T. Nishimura and Y. Sakemi, 'Topological aspect of Wulff shapes', J. Math. Soc. Japan 66 (2014), 89-109.

[16] A. Padrol and G. M. Ziegler, 'Six topics on inscribable polytopes', in Advances in Discrete Differential Geometry (Springer, New York, 2016), 407-419.

[17] R. T. Rockafellar, Convex Analysis (Princeton University Press, Princeton, NJ, 1970).

[18] G. M. Ziegler, Lectures on Polytopes, Graduate Texts in Mathematics, 152 (Springer, New York, 1995). 